\title{
Bore formation, evolution and disintegration into solitons in shallow inhomogeneous channels
}

\author{
J.-G. Caputo ${ }^{1,2}$ and Y. A. Stepanyants ${ }^{3}$ \\ ${ }^{1}$ Laboratoire de Mathématiques, INSA de Rouen, B. P. 8, 76131 Mont-Saint-Aignan cedex, France \\ ${ }^{2}$ Laboratoire de Physique théorique et modelisation, Université de Cergy-Pontoise and C. N. R. S, France \\ ${ }^{3}$ Environment at ANSTO, PMB 1, Menai (Sydney), 2234, NSW, Australia
}

Received: 22 May 2002 - Revised: 19 November 2002 - Accepted: 28 November 2002

\begin{abstract}
The propagation of nonlinear surface waves in channels of smoothly variable in space cross section is studied theoretically and by means of numerical computations. The mathematical model describing wave evolution is based on the generalized Korteweg-de Vries equation with additional terms due to spatial inhomogeneity and energy dissipation. Specifically we consider channels of variable depth and width. The breaking of Riemann waves and the disintegration of hydraulic jumps into trains of solitons have been examined. The results obtained can be useful in particular for the understanding some peculiarities of bore (mascaret) formation, viscous evolution and disintegration into solitons in inhomogeneous channels or rivers.
\end{abstract}

\section{Introduction}

The problem of shallow water wave propagation in rivers, channels or canals has been a matter of interest for at least the two last centuries. John Scott Russell apparently was the first who observed solitary wave propagations in the Union Canal near Edinburgh in 1838 Ablowitz and Segur (1981); Whitham (1974). As an observant researcher, he described in his first Report not only the fact itself of the solitary wave existence ("well defined heap of water ... over a foot hight and thirty feet long") but also its gradual decay due to viscosity and structural stability against the channel inhomogeneity and curvature. Since that first observation many interesting and important results have been obtained. There is no room here to mention all the relevant publications dealing with the problem, therefore we only refer to the most appropriate articles. Despite significant progress in the understanding of the dynamics of surface waves in rivers and channels many problems of practical interest still remain unsolved.

Attempts to apply classical results of shallow water theory to real situations meet with serious difficulties related to the necessity to take into account wave dissipation, boundary

Correspondence to: J.-G. Caputo (caputo@insa-rouen.fr) conditions at the riverbed of a complex cross section, river bending, variability of the river depth and width, etc. All these factors lead to a further development of classical shallow water models, described by Boussinesq, Saint Venant or Korteweg-de Vries equations (KdV), which become nonintegrable. The role of approximate methods and numerical modelling increases significantly in such situations.

In this article we consider the problem of shallow water wave propagation in a channel of smoothly variable in space cross section. We assume a channel of rectangular cross section and take into account variations of its depth or width, its bending and wave dissipation. In Sect. 2 we introduce the main model equation which is the generalized $\mathrm{KdV}$ equation containing additional terms describing the above mentioned effects. Some properties of the model equation are discussed. In Sect. 3 we study the breaking of Riemann waves due to the nonlinearity. The influence of different types of viscous losses in channels of linearly varying depth and width are taken into account. In Sect. 4 we consider the formation of solitons from an initial hydraulic jump due to the effect of dispersion. In particular, we study the dependence of the distance of first soliton emergence from a step-wise perturbation on the amplitude of the perturbation and the depth of the channel. Section 5 is devoted to study the formation of a soliton from small initial disturbances in inhomogeneous channels. In Sect. 6 we present soliton-amplitude decay laws for different types of dissipation. The numerical results, given in Sect. 7, illustrate the evolution of the bore into a train of solitons in a channel of variable depth or width and the influence of different types of dissipations. We present our conclusions in Sect. 8.

\section{The model}

Let us consider long water waves of relatively small amplitude in a channel of rectangular cross section. The KdV model equation describing such perturbations can be derived from the Boussinesq equations of shallow water theory 
Whitham (1974). We will not repeat here the well-known derivation of the equation and one can see for example the careful calculation done by Das Das (1985) for the case of a uniform channel of arbitrary cross section. We will modify this equation to describe waves in a channel whose section varies smoothly in space (gradually varying depth or/and width) and consider simple damping terms. In addition, we assume that the channel may smoothly bend in space. To take this into account, we use a curvilinear coordinate frame so that the undisturbed free surface is taken as the $z=0$ plane with the axis $z$ directed vertically and intersecting the channel bottom. The axis $x$ is directed along the channel and may smoothly bend in space (it is so-called the ray coordinate - the distance along the ray from some fixed point). The axis $y$ is directed across the channel perpendicular both to the axes $x$ and $z$. The axes $x, y$ and $z$ form an orthogonal coordinate frame at each point in space.

Let $h(x)$ be the unperturbed depth of the channel at the equilibrium and $l(x)$ - the width of the channel. We assume there is no mean flow in the channel so that the water is at rest in the absence of perturbations.

\subsection{Generalized KdV equation for the boundary Cauchy} problem

We first consider the classical KdV equation for a rectilinear channel of rectangular cross section, uniform in space (see for instance Das, 1985; Kakutani and Matsuuchi, 1975; Miles, 1975, as well as Ablowitz and Segur, 1981):

$$
\frac{\partial \eta}{\partial t}+\left(c_{0}+a_{1} \eta\right) \frac{\partial \eta}{\partial x}+a_{2} \frac{\partial^{3} \eta}{\partial x^{3}}=0
$$

where $\eta(t, x)$ is the perturbation of the water surface; $c_{0}=$ $\sqrt{g h}$ is the velocity of long linear waves, $g$ is the acceleration due to gravity; $a_{1}=3 c_{0} /(2 h) ; \quad a_{2}=c_{0} h^{2} / 6$.

In the present form Eq. (1) is suitable for the solution of an initial Cauchy problem, i.e. it is supposed that water elevation $\eta$ is known everywhere in space at a given instant of time, $\eta(0, x)=\Phi(x)$, where $\Phi(x)$ is a given function. In such a case one can calculate the further evolution in time of the initial perturbation by means of Eq. (1). This approach is usually used in the mathematical study of Eq. (1) and related equations Ablowitz and Segur (1981).

In practice however, researchers usually deal with measurements of time series of water elevations at some fixed points $\eta\left(t, x_{i}\right)$ and want to predict the further spatial development of the perturbation propagating along the channel if it is known as a function of time, at the boundary of the domain of interest (see, e.g. Osborne, 1995). The corresponding mathematical problem can be called a boundary Cauchy problem for the $\mathrm{KdV}$ equation presented in signalling coordinates (see below). So, we assume that the water elevation is a given function of time at the boundary $x=0: \eta(t, 0)=\Phi(t)$ while at $x>0$ the elevation is zero (the water surface is unperturbed), and aim to predict its further evolution in space by means of the appropriate modification of Eq. (1).
Note, that as was shown in Merchant and Smyth (1991, 2002) the initial-boundary problem described above can be considered, in principle, within the framework of Eq. (1). But such a problem statement is more difficult, in our opinion, than the consideration of a boundary Cauchy problem within the signalling coordinates. Only relatively simple solutions for the initial-boundary problem were obtained so far by means of approximate asymptotic method Merchant and Smyth $(1991,2002)$, while in the signalling coordinates the entire powerful kit of exact methods (including inverse scattering transform, Hirota method, Darboux and Bäclund transformations, etc.) can be used. Note also that the accuracy of the $\mathrm{KdV}$ equation in the traditional form (1) and in the signalling coordinates is the same. Therefore, it is quite reasonable to use the most simple and advanced approach based on the $\mathrm{KdV}$ equation in signalling coordinates.

To obtain the suitable modification of the KdV equation, let us first rewrite Eq. (1) in the following equivalent form:

$\frac{\partial \eta}{\partial x}+\frac{1}{c_{0}} \frac{\partial \eta}{\partial t}+\frac{a_{1}}{c_{0}} \eta \frac{\partial \eta}{\partial x}+\frac{a_{2}}{c_{0}} \frac{\partial^{3} \eta}{\partial x^{3}}=0$.

Then, we point out that the derivation of KdV equation assumes that in the zero-order approximation wave processes obey the simple wave equation

$\frac{\partial \eta}{\partial t}+c_{0} \frac{\partial \eta}{\partial x}=0$

and all other effects (nonlinearity, dispersion, dissipation, etc.) appear as small corrections to this equation in the next approximations. This implies that the following relationship between derivatives

$\frac{\partial}{\partial x}=-\frac{1}{c_{0}} \frac{\partial}{\partial t}$

which follows from Eq. (3), can be used for the higher-order approximations. Hence, one can replace the spatial derivatives by time derivatives in the nonlinear $\left(\sim a_{1}\right)$ and dispersive $\left(\sim a_{2}\right)$ terms. As a result, Eq. (1) takes the following form in the signalling coordinates (cf. Osborne, 1995)

$\frac{\partial \eta}{\partial x}+\frac{1}{c_{0}} \frac{\partial \eta}{\partial t}-\frac{a_{1}}{c_{0}^{2}} \eta \frac{\partial \eta}{\partial t}-\frac{a_{2}}{c_{0}^{4}} \frac{\partial^{3} \eta}{\partial t^{3}}=0$.

This equation allows a further generalization. First of all, the effect of the channel inhomogeneity can be taken into account. This effect is important from the practical point of view because natural channels or rivers are never rectilinear in reality but rather curved. However, if the channel bend is smooth enough, i.e. $\lambda / R \ll 1$, where $\lambda$ is a characteristic wave length and $R$ is the curvature radius of a channel, then Eq. (5) is still applicable, but the axis $x$ is no more a Cartesian one. It must be replaced by the curvilinear "ray" coordinate directed along the channel axis orthogonal at each of its point to the other two axes, $y$ and $z$ (Fig. 1). A similar approach was used for the description of sea waves in a coastal zone with a nonuniform bottom which results in the formation of curvilinear waveguides Pelinovsky et al. (1993). 


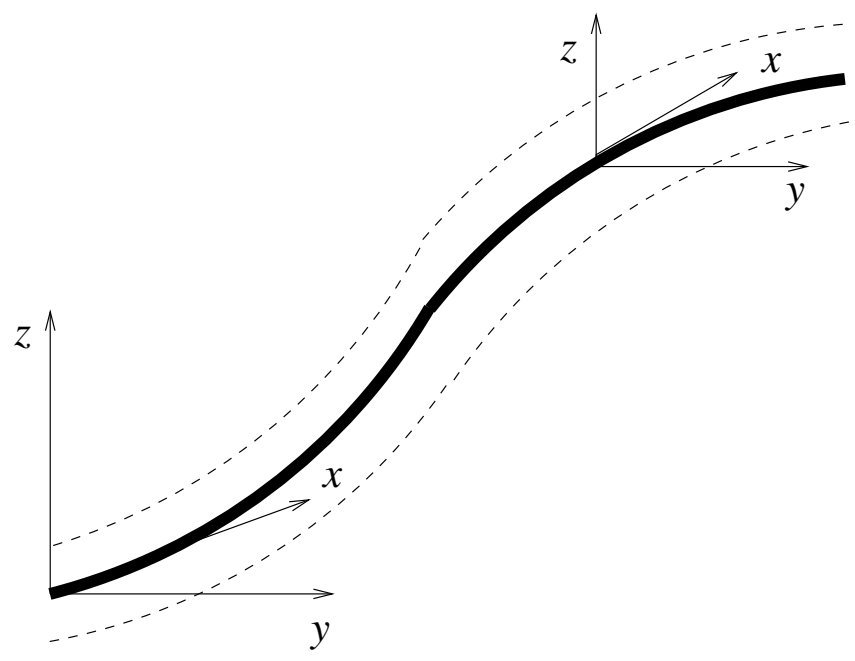

Fig. 1. Sketch of a channel bend and a curvilinear coordinate frame.

The next, more important point, is the variability in space of the channel parameters. The depth $h$, channel width $l$, wave speed $c_{0}$, etc. are not constants in general, but depend on the spatial ray coordinate $x$. This leads not only to an $x$-dependence of the coefficients of the Eq. (5) but also to the appearance of an additional term. To derive this term, we note first that the constructing generalized $\mathrm{KdV}$ equation contains additively the terms describing different effects such as nonlinearity, dispersion, dissipation, inhomogeneity, etc, because all these effects are assumed small and the same order of smallness. This assumption allows us to derive the corresponding terms in the equation separately from others. The key point to derive the inhomogeneous term is the law of energy flux conservation across the channel cross sectional area. Time averaged density of wave energy integrated over the channel depth can be readily obtained in the linear approximation $\mathcal{E}=\rho g \eta^{2} / 2$ Mei (1983); Stepanyants (1985), where $\rho$ is fluid density. Hence, the total energy flux across the channel cross section is

$Q=c_{0}(x) \mathcal{E} l(x)=1 / 2 \rho g c_{0}(x) l(x) \eta^{2}(x)=$ const.

By differentiating Eq. (6) over $x$ one obtains

$\frac{\partial \eta}{\partial x}+\frac{\eta}{2 c_{0} l} \frac{\partial\left(c_{0} l\right)}{\partial x}=0$.

This equation describes wave amplitude variation in a channel of variable cross section, wave amplitude can vary in space both due to variation of the channel width, $l(x)$, and due to variation of channel depth because $c_{0}(x)=\sqrt{g h(x)}$.

We assume that the channel inhomogeneity is smooth enough so that the second term in Eq. (7) is of the same order of smallness as nonlinear and dispersive terms in Eq. (5) and, hence, it can be included into that Eq. (5) additively. Of course, a strict mathematical derivation of such a generalized $\mathrm{KdV}$ equation can be done on the basis of a regular asymptotic method (see, e.g. Ostrovsky, 1974; Pelinovsky, 1996) but it is beyond the scope of this article. Actually, such sort of equations are very well-known already for a long time (see, e.g. Grimshaw, 1970; Ostrovsky and Pelinovsky, 1970), and we just remind the reader how different physical effects give rise to separate terms in the generalized KdV Eq. (8) written below.

In addition to the inhomogeneity effect, we also take into account a small dissipation assuming again that the corresponding term in the generalized $\mathrm{KdV}$ equation is of the same order of smallness as other small terms. We present dissipation by some generalized function $F(\eta)$ in the right-hand side of the model equation, leaving the discussion of a possible structure of the corresponding viscous term to the next subsection.

So, the combination of all effects mentioned above leads eventually to the following model equation

$$
\begin{aligned}
\frac{\partial \eta}{\partial x} & +\frac{1}{c_{0}(x)}[1-\alpha(x) \eta] \frac{\partial \eta}{\partial t}-\beta(x) \frac{\partial^{3} \eta}{\partial t^{3}} \\
& +\frac{\eta}{2 \Delta(x)} \frac{d \Delta(x)}{d x}=-F(\eta),
\end{aligned}
$$

where

$$
\begin{aligned}
& \alpha(x)=\frac{a_{1}(x)}{c_{0}(x)}=\frac{3}{2 h(x)}, \quad \beta=\frac{a_{2}(x)}{c_{0}^{4}(x)}=\frac{h^{2}(x)}{6 c_{0}^{3}(x)} ; \\
& \Delta(x)=c_{0}(x) l(x) .
\end{aligned}
$$

The function $\Delta(x)$ is the analog of the ray divergence factor in the theory of sea waves in an inhomogeneous coastal zone Pelinovsky (1996). An equation similar to Eq. (8) including inhomogeneity and viscous terms has been strictly derived in Grimshaw (1981) for long nonlinear internal waves in stratified shear flows. Surface waves can be considered as a limiting case of internal waves in a two-layer fluid with a sharp density interface and a zero density upper layer.

Wave dynamics in channels or rivers essentially depends also on the shape of initial perturbations (we are using the term "initial" but in fact we consider perturbations given at a fixed point $x_{0}$ as was already pointed out). Other important factors are the amplitude and duration of the perturbation as well as external parameters determining the coefficients of a generalized KdV Eq. (8). Unfortunately, Eq. (8) is too complex to analyze its solutions in general, therefore we consider some most typical and important wave processes which can occur in the natural condition; these processes we will study here in detail.

\subsection{Models of dissipation}

There are several different models of dissipation, unfortunately none of them has been thoroughly examined experimentally for surface waves. There are a few articles where dissipation of long nonlinear waves was studied experimentally in laboratory conditions (see about that in Ablowitz and Segur, 1981) but the applicability of the results obtained to natural conditions is doubtful. The most frequently used models for practical estimations of wave decay are listed in this subsection. 
In the simplest case, the dissipative term in the right-hand side of Eq. (8) is frequency independent, we will call this model the Rayleigh dissipation Pelinovsky et al. (1993):

$$
F(\eta)=\gamma_{0}(x) \eta, \quad \text { where } \quad \gamma_{0}(x)=\frac{3 v}{4 c_{0}(x) h^{2}(x)},
$$

and $v\left(\mathrm{~m}^{2} / \mathrm{s}\right)$ is the turbulent viscosity of water which is usually an empirical parameter. It varies in a wide range from 0.2 to $30 \mathrm{~m}^{2} / \mathrm{s}$ Holloway et al. (1997), while the usual molecular viscosity is only $10^{-6} \mathrm{~m}^{2} / \mathrm{s}$. Essentially the same dissipation in shallow water has been recently considered in $\mathrm{Wu}$ and Tian (2000).

A more complex case corresponds to Chezy model of wave dissipation Pelinovsky et al. (1993); Holloway et al. (1997, 1999):

$$
F(\eta)=\gamma_{c}(x)|\eta| \eta, \quad \text { where } \quad \gamma_{c}(x)=\frac{k}{h^{2}(x)},
$$

$k \approx 1.5 \cdot 10^{-3}$ is an empirical dimensionless coefficient describing friction on the bottom. Equations containing this type of losses have been widely used to describe stationary flows in river hydraulics and the dynamics of wave breaking in a surf zone (see, e.g. Leontjev, 1989; Voltsinger et al., 1989, and references therein).

Sometimes a dissipation of the Reynolds type is considered with some effective turbulent viscosity instead of a molecular one. This leads to the appearing of Burgers viscous term in the generalized KdV Eq. (8) (Holloway et al., 1997):

$F(\eta)=-\gamma_{2}(x) \frac{\partial^{2} \eta}{\partial t^{2}}, \quad$ where $\quad \gamma_{2}(x)=\frac{\nu}{c_{0}^{3}}$

and $v\left(\mathrm{~m}^{2} / \mathrm{s}\right)$ is again an empirical coefficient of effective turbulent viscosity as above.

It is interesting to note that the same term describes energy losses by linear surface waves due to scattering on bottom roughness (Dyatlov and Pelinovsky, 1990). The dissipative coefficient can be calculated analytically in this case:

$\gamma_{2}=\frac{\Lambda}{c_{0}^{2}(x)}\left[\frac{a_{a v}}{h(x)}\right]^{2}$,

where $a_{a v}$ is the average height of bottom roughness and $\Lambda$ is the characteristic scale of irregularities (both these parameters can be $x$-dependent in principle). Unfortunately, this model is not applicable to the description of nonlinear wave scattering (Benilov and Pelinovsky, 1988), and currently there is no simple modification for this case.

The most rigorous study of energy losses caused by water friction on the channel bottom and side walls leads to a $\mathrm{KdV}$ equation with the integral viscous term in the form (Das, 1985; Kakutani and Matsuuchi, 1975; Miles, 1975)

$$
\begin{aligned}
F(\eta)= & \frac{a_{3}}{4 c_{0} \sqrt{\pi c_{0} \operatorname{Re}}} \\
& \cdot \int_{-\infty}^{+\infty} \frac{1-\operatorname{sgn}\left(t-t^{\prime}\right)}{\sqrt{\left|t-t^{\prime}\right|}} \frac{\partial \eta\left(t^{\prime}, x\right)}{\partial t^{\prime}} d t^{\prime} .
\end{aligned}
$$

A similar viscous term has been derived in Grimshaw (1981) for internal waves in shear flow. Actually this term combines dissipative and dispersive features. The expression for the coefficient $a_{3}$ can be found in Kakutani and Matsuuchi (1975) and Miles (1975) for a channel of rectangular cross section and in Das (1985) for a channel of arbitrary cross section. Despite the fact that the derivation of this term seems to be the most accurate, its applicability to real natural or laboratory situations of water wave decay has not been confirmed yet either experimentally or numerically. In addition, this integral term makes the $\mathrm{KdV}$ equation difficult to analyze in general, meanwhile the approximate solutions describing smooth decay of solitary and periodic waves in a homogeneous channel have been obtained in Kakutani and Matsuuchi (1975) and Miles (1976). We will not consider this type of dissipation in the present article, it is mentioned here just for the completeness.

\subsection{Conservation laws}

Equation (8) does not belong to the class of completely integrable models even in the absence of dissipation. However, in the dissipationless case, when $F(\eta)=0$, it possesses at least two integrals of motion.

The first one corresponds to the mass conservation in the homogeneous medium and can be treated as conservation of the mass flux in the inhomogeneous case (this integral of motion remains valid when Burgers viscosity is included):

$\Delta^{1 / 2}(x) \int \eta d t=$ const .

The integral is taken over a wave period for periodical perturbations or from minus to plus infinity for solitary perturbations vanishing at the infinity.

The second conserved quantity corresponds to the energy conservation in the homogeneous medium whereas in the inhomogeneous case it can be treated as conservation of the energy flux:

$\Delta(x) \int \eta^{2} d t=$ const.

From these conservation laws, one can estimate how the amplitude of a given initial perturbation varies in space. In particular, a linear dispersionless perturbation has a duration $T$ which is independent of its amplitude $A$. In this case both these integrals give the same law of wave amplitude variation with distance if the depth and width of the channel are known. Consider for example a perturbation

$\eta(x, t)=A f(\tau / T), \quad \tau=t-\int_{0}^{x} \frac{d x^{\prime}}{c_{0}\left(x^{\prime}\right)}$,

where $f$ is a dimensionless function describing wave profile. Substituting this expression into one of the above integrals, (15) or (16), say into (16) for certainty, one obtains

$\Delta(x) A^{2}(x) T \int f^{2}(\theta) d \theta=$ const . 
It readily follows then

$A(x) \sim \Delta^{-1 / 2}(x) \sim h^{-1 / 4}(x) l^{-1 / 2}(x)$.

The well-known Green's law, $A(x) \sim h^{-1 / 4}(x)$, follows from this formula in the particular case of a channel of a constant width. The dependence of wave amplitude on the channel width at a constant depth is more strong than on the depth, $A(x) \sim l^{-1 / 2}(x)$.

In the nonlinear case the situation turns to be a bit more complex because wave duration and amplitude are usually linked with each other. For a KdV soliton of the form

$\eta(x, t)=A \operatorname{sech}^{2}\left[\frac{1}{-T}\left(t-\int_{0}^{x} \frac{d x^{\prime}}{V\left(x^{\prime}\right)}\right)\right]$

there are relationships between the amplitude and duration (see, e.g. Ablowitz and Segur, 1981; Karpman, 1973; Whitham, 1974), $T=\sqrt{12 c_{0} \beta / \alpha A}$, and between the amplitude and wave velocity,

$V=c_{0} /(1-\alpha A / 3) \approx c_{0}(1+\alpha A / 3)$.

In the nonlinear case only the second integral of motion (16) gives the correct formula for soliton amplitude variation with distance. Strictly speaking this dependence must be derived by successive application of the asymptotic method (see, e.g. Ostrovsky, 1974) based on the smallness of the inhomogeneity, the characteristic width of a soliton is assumed to be small in comparison with the scale of the inhomogeneity. However, a long and tedious asymptotic procedure leads to the same result as follows from the variational approach using Eq. (16) but not Eq. (15). The reason is that as it evolves in an inhomogeneous medium the soliton is gradually destroyed and generates behind itself a nonsoliton perturbation in the form of a shelf. This perturbation apparently contributes significantly to the mass flux (15) but not so much to the energy flux (16). Therefore, by substituting the soliton expression (20) into Eq. (16) and neglecting the shelf, one obtains instead of Eq. (18)

$$
\begin{aligned}
& \Delta(x) A^{2}(x) T(x) \int_{-\infty}^{+\infty} \operatorname{sech}^{4}(\theta) d \theta \\
& \quad=\Delta(x) A^{3 / 2}(x) \sqrt{\frac{c_{0}(x) \beta(x)}{\alpha(x)}}=\text { const } .
\end{aligned}
$$

Using the expressions (9) for the coefficients of the $\mathrm{KdV}$ equation, one readily obtains

$l(x) h^{3 / 2}(x) A^{3 / 2}(x)=$ const

or

$A(x) \sim l^{-2 / 3}(x) h^{-1}(x)$.

For channels of variable depth and constant width this implies a nonlinear analog of Green's law $A(x) \sim$ $h^{-1}(x), T(x) \sim h^{3 / 2}(x)$ (see Ostrovsky and Pelinovsky, 1970, 1975, where generalized Green's laws were obtained both for solitary and for periodic perturbations). When the width of the channel varies and the depth is constant, Eq. (22) gives $A(x) \sim l^{-2 / 3}(x), T(x) \sim l^{1 / 3}(x)$.

\subsection{Simplification of the main Eq. (8)}

First of all, let us exclude the inhomogeneous term from Eq. (8) using the transformation:

$u(x, t)=s(x) \eta(x, t), \quad$ where $\quad s(x)=\sqrt{\frac{\Delta(x)}{\Delta(0)}}$.

The resultant equation takes the form:

$$
\begin{aligned}
\frac{\partial u}{\partial x} & +\frac{1}{c_{0}(x)}\left[1-\frac{\alpha(x)}{s(x)} u\right] \frac{\partial u}{\partial t}-\beta(x) \frac{\partial^{3} u}{\partial t^{3}} \\
& =-s(x) F\left[\frac{u}{s(x)}\right] .
\end{aligned}
$$

As a next step, one can remove the linear term in the above equation using the transformation of independent variables:

$\tau=t-\int_{0}^{x} \frac{d x^{\prime}}{c_{0}\left(x^{\prime}\right)}, \quad \xi=x$.

The equation takes the form

$\frac{\partial u}{\partial \xi}-p(\xi) u \frac{\partial u}{\partial \tau}-\beta(\xi) \frac{\partial^{3} u}{\partial \tau^{3}}=-\mathcal{F}(u)$.

where

$p(\xi)=\frac{\alpha(\xi)}{c_{0}(\xi) s(\xi)}, \quad \mathcal{F}(u)=s(\xi) F\left[\frac{u}{s(\xi)}\right]$.

Note, that if the function $F(u)$ is linear (which is the case, e.g. of Rayleigh or Reynolds dissipation), then $\mathcal{F}(u) \equiv$ $F(u)$.

Further simplification can be made if one considers a boundary condition in a two-parametric form (note that $u(0, \tau) \equiv \eta(0, t))$ :

$u(0, \tau)=U \Phi(\tau / T)$

where $U$ is a characteristic amplitude of the "initial" perturbation, $T$ is its characteristic duration and $\Phi$ is a dimensionless function describing the shape of the perturbation given at the boundary $x=0$.

Making the new transformation

$\zeta=\frac{U}{T} \int_{0}^{\xi} p\left(\xi^{\prime}\right) d \xi^{\prime}, \quad \theta=\frac{\tau}{T}, \quad v=\frac{u}{U}$,

one can reduce Eq. (26) to the dimensionless form:

$\frac{\partial v}{\partial \zeta}-v \frac{\partial v}{\partial \theta}-\frac{1}{\sigma^{2}(\zeta)} \frac{\partial^{3} v}{\partial \theta^{3}}=-\frac{1}{R_{d}} \mathcal{F}(v)$.

where $\sigma^{2}(\zeta)=p(\zeta) U T^{2} / \beta(\zeta)$ is the Ursell's parameter, $R_{d}=p(\zeta) U / T$ is the dissipation parameter (the analog of Reynolds number in hydrodynamics), and function $\mathcal{F}(v)$ is given by one of the three kinds of dissipations mentioned above, Eqs. (10)-(12), or by their combinations:

$$
\begin{aligned}
& \mathcal{F}(v)= \\
& \left\{\frac{3 v}{4 c_{0}(\zeta) h^{2}(\zeta)} v ; \frac{k U}{s(\zeta) h^{2}(\zeta)}|v| v \frac{v}{T^{2} c_{0}^{3}(\zeta)} \frac{\partial^{2} v}{\partial \theta^{2}}\right\} .
\end{aligned}
$$


Table 1. Dependence of the different damping coefficients on $\zeta$ for a channel of linearly decreasing depth

\begin{tabular}{lll}
\hline $\begin{array}{c}\text { Type of } \\
\text { dissipation }\end{array}$ & \multicolumn{1}{c}{ Coefficient } & \multicolumn{1}{c}{$\begin{array}{c}\text { Normalized } \\
\text { coefficient }\end{array}$} \\
\hline Rayleigh & $g_{0} \equiv \frac{1}{R_{d}} \frac{3 v}{4 c_{0}(\zeta) h^{2}(\zeta)}=\frac{v T}{2 U h_{0}} g_{0}^{h}(\zeta)$ & $g_{0}^{h}(\zeta)=1+W_{h} \zeta$ \\
Chezy & $g_{c} \equiv \frac{1}{R_{d}} \frac{k U}{s(\zeta) h^{2}(\zeta)}=\frac{2}{3} \sqrt{\frac{g}{h_{0}}} T k g_{c}^{h}(\zeta)$ & $g_{c}^{h}(\zeta)=\left(1+W_{h} \zeta\right)^{2 / 3}$ \\
Reynolds & $g_{2} \equiv \frac{1}{R_{d}} \frac{v}{T^{2} c_{0}^{3}(\zeta)}=\frac{2}{3} \frac{v}{U T g} g_{2}^{h}(\zeta)$ & $g_{2}^{h}(\zeta)=\left(1+W_{h} \zeta\right)^{-1 / 3}$ \\
\hline
\end{tabular}

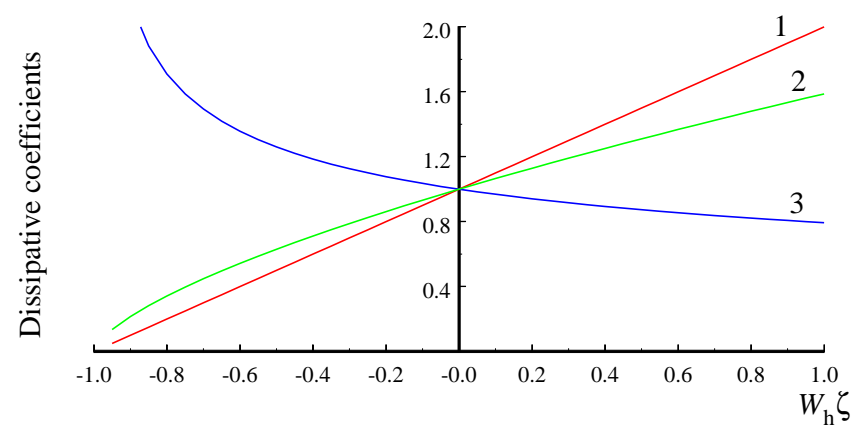

Fig. 2. Dependences of the normalized dissipative coefficients $g_{0}^{h}(\zeta), g_{c}^{h}(\zeta)$ and $g_{2}^{h}(\zeta)$ labeled respectively 1,2 and 3 , on the normalized distance $W_{h} \zeta$ for a channel of variable depth.

\subsubsection{Relative effectiveness of different types of dissipations}

Let us compare the relative effectiveness of the different types of dissipations in an inhomogeneous channel. Suppose first that the depth of the channel decreases linearly:

$h(x)=h_{0}\left(1-\kappa_{h} x\right), \quad$ where $\quad 0 \leq x \leq X_{o r} \equiv 1 / \kappa_{h}$,

while its width remains constant. In this case, one can calculate the different dissipative terms in the right-hand side of Eq. (29) with $\mathcal{F}(v)$ given by Eq. (30). The corresponding dissipation coefficients $g_{0}, g_{c}$ and $g_{2}$ are presented in Table 1 in the normalized form as functions of the variable $W_{h} \zeta$ where $W_{h}=\kappa_{h} T \sqrt{g h_{0}^{3}} /(2 U)$.

The dependences of the above defined normalized coefficients $g_{0}^{h}(\zeta), g_{c}^{h}(\zeta)$ and $g_{2}^{h}(\zeta)$ on $\zeta$ are shown in Fig. 2, respectively as curves 1,2 and 3 .

As one can see, the coefficients of Rayleigh and Chezy dissipation increase with distance as the wave propagates towards the shallower part of the channel, while the coefficient of Reynolds viscosity decreases. Note also that the total contribution of each dissipative term depends not only on the values of the coefficients but also on the local wave parameters (amplitude and duration in general) so that the relative strength of the different dissipative terms can vary differently. As an example, let us estimate the ratio of Reynolds to Rayleigh dissipations for a $\mathrm{KdV}$ soliton in a channel of variable depth:

$$
\begin{aligned}
\frac{\text { Reynolds dissipation }}{\text { Rayleigh dissipation }} & =\frac{v}{T^{2} c_{0}^{3}(\zeta)} \frac{\partial^{2} v}{\partial \theta^{2}}: \frac{3 v v}{4 c_{0}(\zeta) h^{2}(\zeta)} \\
& =\frac{4}{3} \frac{h(\zeta)}{g T^{2}} \frac{1}{v} \frac{\partial^{2} v}{\partial \theta^{2}} \approx \frac{4}{3} \frac{h(\zeta)}{g T^{2} \mathcal{T}^{2}(\zeta)},
\end{aligned}
$$

where $\mathcal{T}(\zeta)$ is the soliton duration in dimensionless variables. Taking into consideration the "nonlinear Green's law" for $\mathrm{KdV}$ solitons (see Sect. 2.3) and the relationship between the soliton amplitude $\mathcal{A}$ and its duration which in the dimensionless variables is

$\mathcal{T}^{2}(\zeta)=\frac{12}{\sigma^{2}(\zeta) \mathcal{A}(\zeta)}=\frac{4 h_{0}^{2}}{3 g T^{2} U}\left(1+W_{h} \zeta\right)^{-13 / 3}$,

one finally obtains

$\frac{\text { Reynolds dissipation }}{\text { Rayleigh dissipation }}=\frac{U}{h_{0}}\left(1+W_{h} \zeta\right)^{3}$.

Hence, the relative strength of the Reynolds dissipation with respect to the Rayleigh one smoothly increases with distance for a $\mathrm{KdV}$ soliton.

Note that the Ursell dispersion parameter increases in the same proportion

$\sigma^{2}(\zeta)=\frac{9 g U T^{2}}{h_{0}^{2}}\left(1+W_{h} \zeta\right)^{3}$.

Let us suppose now that the width of the channel decreases linearly:

$l(x)=l_{0}\left(1-\kappa_{l} x\right), \quad$ where $0 \leq x \leq X_{o r} \equiv 1 / \kappa_{l}$,

while the depth remains constant. Using again the formulae above and denoting $W_{l}=T c_{0} h \kappa_{l} /(2 U)$, the same term as $W_{h}$, one obtains the dependences given in Table 2 .

The normalized coefficients $g_{0}^{l}(\zeta), \quad g_{c}^{l}$ and $g_{2}^{l}(\zeta)$ are shown in Fig. 3 as curves 1, 2 and 3 respectively.

The coefficients of Rayleigh and Reynolds dissipation decrease with distance as the wave propagates towards the narrower part of the channel, while the coefficient of Chezy dissipation remains constant. The ratio of Reynolds to Rayleigh 
Table 2. Dependence of the different damping coefficients on $\zeta$ for a channel of linearly decreasing width

\begin{tabular}{llc}
\hline $\begin{array}{c}\text { Type of } \\
\text { dissipation }\end{array}$ & Coefficient & $\begin{array}{c}\text { Normalized } \\
\text { coefficient }\end{array}$ \\
\hline Rayleigh & $g_{0} \equiv \frac{1}{R_{d}} \frac{3 v}{4 c_{0} h^{2}}=\frac{3}{4} \frac{T}{p(\zeta) U} \frac{v}{c_{0} h^{2}}=\frac{v T}{2 U h} g_{0}^{l}(\zeta)$ & $g_{0}^{l}(\zeta)=1-\frac{2}{3} W_{l} \zeta$ \\
Chezy & $g_{c} \equiv \frac{1}{R_{d}} \frac{k U}{s(\zeta) h^{2}}=\frac{2}{3} \sqrt{\frac{g}{h}} T k g_{c}^{l}(\zeta)$ & $g_{c}^{l}(\zeta)=1$ \\
Reynolds & $g_{2} \equiv \frac{1}{R_{d}} \frac{v}{T^{2} c_{0}^{3}}=\frac{v}{U T p(\zeta) c_{0}^{3}}=\frac{2}{3} \frac{v}{U T g} g_{2}^{l}(\zeta)$ & $g_{2}^{l}(\zeta)=1-\frac{2}{3} W_{l} \zeta$ \\
\hline
\end{tabular}

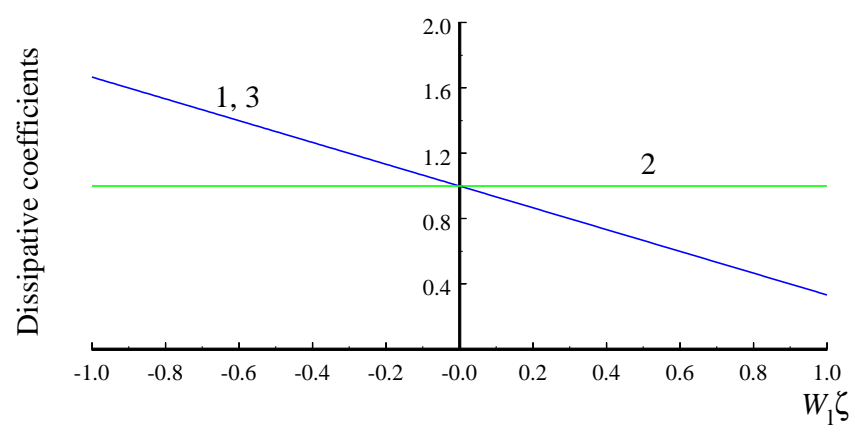

Fig. 3. Dependences of the normalized dissipative coefficients $g_{0}^{l}(\zeta), \quad g_{c}^{l}$ and $g_{2}^{l}(\zeta)$ labeled respectively 1,2 and 3 , on the normalized distance $W_{l} \zeta$ for a channel of variable width.

dissipations for a $\mathrm{KdV}$ soliton in a channel of variable width is:

$$
\begin{aligned}
\frac{\text { Reynolds dissipation }}{\text { Rayleigh dissipation }} & =\frac{2}{3} \frac{v}{g T U} \frac{\partial^{2} v}{\partial \theta^{2}}: \frac{v T v}{2 U h} \\
& =\frac{4}{3} \frac{h}{g T^{2}} \frac{1}{v} \frac{\partial^{2} v}{\partial \theta^{2}} \approx \frac{4}{3} \frac{h}{g T^{2} \mathcal{T}^{2}(\zeta)} .
\end{aligned}
$$

Taking into consideration the "nonlinear Green's law" for $\mathrm{KdV}$ solitons in a channel of variable width (see the end of the Sect. 2.3), one obtains

$\frac{\text { Reynolds dissipation }}{\text { Rayleigh dissipation }}=\frac{U}{h}\left(1-\frac{2}{3} W_{l} \zeta\right)^{-7 / 3}$.

Again the relative strength of the Reynolds dissipation with respect to the Rayleigh one smoothly increases with distance for a KdV soliton. Both dependences (33) and (37), for a channel of variable depth and variable width, respectively, are shown in Fig. 4 (curves 1 and 2). Both these ratios increase as the wave propagates towards the shallower and narrower part of the channel.

The Ursell parameter is given by

$$
\sigma^{2}(\zeta)=\frac{9 g U T^{2}}{h^{2}}\left(1-\frac{2}{3} W_{l} \zeta\right)^{-1}
$$

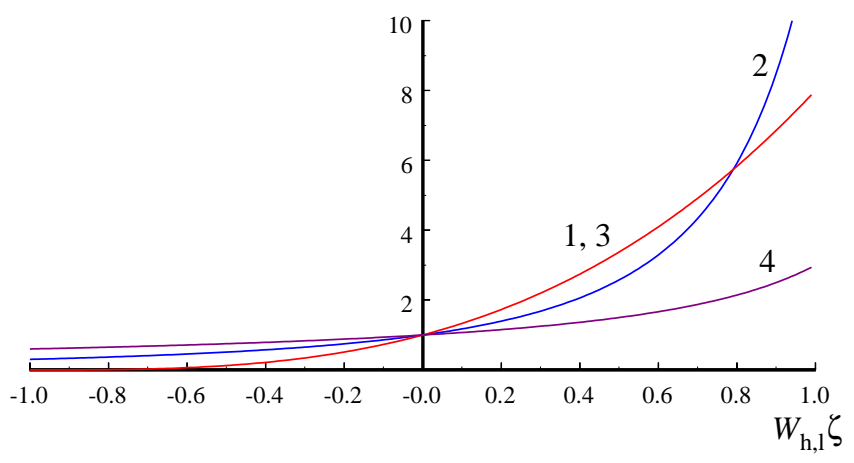

Fig. 4. The ratio of the Reynolds dissipation to the Rayleigh one normalized to $U / h_{0}$ versus the dimensionless distance $W_{h, l} \zeta$ for a KdV soliton propagating in a channel of linearly varying depth (curve 1) and width (curve 2) and analogous dependencies for the normalized Ursell parameters (34) (curve 3) and (38) (curve 4).

The normalized Ursell parameters, $\sigma^{2}(\zeta) h_{0}^{2} /\left(9 g U T^{2}\right)$, are also shown in Fig. 4 for both the variable depth or width cases described by Eqs. (34) and (38) (curves 3 and 4 respectively).

The model Eq. (8) or its dimensionless version (29) is the base for our study of nonlinear waves in an inhomogeneous channel. In the subsequent sections we will analyze it subject to some specific "initial" conditions.

\section{Breaking of Riemann waves}

We consider first very long and smooth perturbations for which dispersive effects are insignificant. This means that the corresponding dispersive term $(\sim \beta)$ in Eq. (24) can be dropped. Let us omit the dissipation for a while. The influence of different types of dissipation on the breaking phenomenon will be briefly discussed at the end of this section. So, Eq. (24) with $\beta=0$ and $F(\eta)=0$ reduces to:

$\frac{\partial u}{\partial x}+\frac{1}{V(u, x)} \frac{\partial u}{\partial t}=0$,

where

$V(u, x)=\frac{c_{0}(x)}{1-\alpha(x) u / s(x)}$. 
Suppose that at $x=0$ the boundary condition is given by a smooth function $\Phi(t)$. An exact solution of Eq. (39) can be presented in the implicit form (Whitham, 1974):

$u(x, t)=\Phi(\tau)$

where

$\tau=t-\int_{0}^{x}\left[1-\frac{\alpha\left(x^{\prime}\right) u\left(x^{\prime}, t\right)}{s\left(x^{\prime}\right)}\right] \frac{d x^{\prime}}{c_{0}\left(x^{\prime}\right)}$.

According to the theory of nonlinear waves (see, e.g. Whitham, 1974), the evolution of a smooth "initial" perturbation described by this solution (41) leads in general to the development of a discontinuity in the wave profile, $u_{t}=u_{x}=\infty$. The distance at which the discontinuity appears, $X_{b}$, is calculated as the minimum root of the transcendental equation (cf. Whitham (1974))

$\frac{\partial}{\partial t}\left(t+\int_{0}^{X_{b}}\left[1-\frac{\alpha(x)}{s(x)} \Phi(t)\right] \frac{d x}{c_{0}(x)}\right)=0$,

which results in

$\int_{0}^{X_{b}} \frac{\alpha(x)}{s(x) c_{0}(x)} d x=M_{d}^{-1}$,

where

$M_{d}=\max \left|\frac{d \Phi(t)}{d t}\right|$.

For a detailed analysis of this formula one should specify the character of the channel inhomogeneity and wave profile at the given boundary.

\subsection{A channel of linearly decreasing depth}

Let us assume that the depth of the channel decreases linearly with $x$ in accordance with Eq. (31), and the channel width $l$ is constant. Then, the parameter $s(x) \sim \sqrt{c_{0}(x)} \sim \sqrt[4]{1-\kappa_{h} x}$, so that from Eq. (43) one obtains

$\frac{3}{2 h_{0} \sqrt{g h_{0}}} \int_{0}^{X_{b}}\left(1-\kappa_{h} x\right)^{-7 / 4} d x=M_{d}^{-1}$.

The integral in Eq. (44) can be readily calculated and the breaking distance $X_{b}$ can be found explicitly

$$
\begin{aligned}
\frac{X_{b}}{X_{0}} & =\frac{1}{\kappa_{h} X_{0}}\left[1-\left(1+\frac{3}{4} \kappa_{h} X_{0}\right)^{-4 / 3}\right] \\
& \approx 1-\frac{7}{8} \kappa_{h} X_{0}
\end{aligned}
$$

where $X_{0}$ is the wave-breaking distance in the homogeneous case when $\kappa_{h}=0$ :

$X_{0}=\frac{2}{3} \frac{h_{0} \sqrt{g h_{0}}}{M_{d}}$.

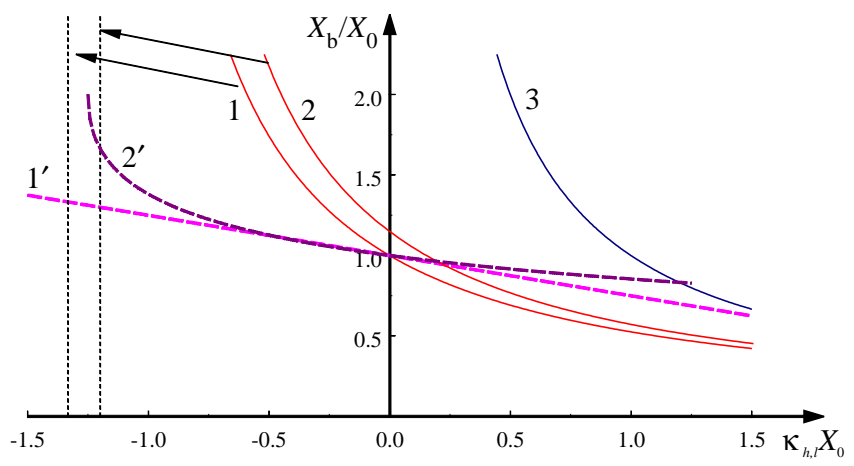

Fig. 5. Dependencies of the normalized breaking distances of a Riemann wave, $X_{b} / X_{0}$, on the normalized gradients of channel depth $\kappa_{h} X_{0}$ (line 1) and channel width $\kappa_{l} X_{0}$ (line $\left.1^{\prime}\right)$. Curve 3 depicts the normalized distance to the channel origin, $X_{o r} / X_{0}$, where its depth or width turns to zero according to the linear models (31), (35). Lines 2 and $2^{\prime}$ show the influence of the Rayleigh dissipation on wave breaking (see Sect. 3.3.1).

Assuming that the perturbation at the boundary $x=0$ is sinusoidal: $\Phi(t)=A \sin \omega t\left(M_{d}=A \omega\right)$ and putting

$A=1 \mathrm{~m}, \quad h_{0}=10 \mathrm{~m}$,

$g=9.8 \mathrm{~m} \mathrm{~s}^{-2}, \quad \omega=0.5 \mathrm{~s}^{-1}$,

one readily obtains that in a channel of uniform depth the breaking distance is $X_{0} \approx 131 \mathrm{~m}$, while in a hypothetic channel of fast decreasing depth, so that $\kappa_{h}=5 \cdot 10^{-3} \mathrm{~m}^{-1}$, this distance is $X_{b} \approx 109 \mathrm{~m}$. In general, $X_{b}$ depends smoothly on $\kappa_{h}$, it decreases monotonically when $\kappa_{h}$ grows (Fig. 5). The breaking distance always remains less than the distance to the origin of the channel $X_{o r}=1 / \kappa_{h}$, where the depth is zero (see Eq. 31). The dependence of $X_{o r}$ on $\kappa_{h}$ is also shown in the same Fig. 5 in normalized variables (see curve 3 ). Only in the limit $\kappa_{h} \rightarrow \infty$ does the breaking distance approach $X_{o r}$. So a decrease of the depth cannot prevent the breaking phenomenon, on the contrary, it even stimulates it because the role of nonlinearity, responsible for the wave breaking, increases when the channel depth decreases.

The situation is different if the channel depth increases. As follows from Eq. (45), $X_{b}$ infinitely increases when $\kappa_{h}$ is negative and approaches the critical value $\kappa_{c r}=-\frac{4}{3} X_{0}^{-1}$. For the sinusoidal perturbation with parameters (47), $\kappa_{c r}=$ $-0.01 \mathrm{~m}^{-1}$. Wave breaking is impossible if the depth of the channel increases too fast so that the depth gradient $\kappa_{h}<\kappa_{c r}$.

\subsection{A channel of linearly decreasing width}

Similar formulae can be derived for a channel of linearly decreasing width, Eq. (35), where depth is assumed constant. By substitution of $l(x)$ in Eq. (43) and simple manipulations one can readily obtain

$\frac{X_{b}}{X_{0}}=\frac{1}{\kappa_{l} X_{0}}\left[1-\left(1-\frac{\kappa_{l} X_{0}}{2}\right)^{2}\right]=1-\frac{\kappa_{l} X_{0}}{4}$.

In the limiting case $\kappa_{l}=0$ Eq. (48) yields again the expression for the homogeneous channel, given by Eq. (46). 
The dependence of $X_{b}$ on $\kappa_{l}$ is linear and weaker than on $\kappa_{h}$ (compare lines 1 and $1^{\prime}$ in Fig. 5). In particluar, $X_{b}$ is smaller in this case than for a variable width. For the parameters considered in the previous section we get $X_{b}=31 \mathrm{~m}$.

Wave breaking always formally occurs for negative $\kappa_{l}$ but the breaking distance increases to infinity for large negative $\kappa_{l}$. For positive $\kappa_{l}$, the breaking distance is always less than the distance to the channel origin, $X_{o r}$, and both these values coincide when $\kappa_{l} X_{0}=2$ (lines $1^{\prime}$ and 3 in Fig. 5 cross at this point). For the sinusoidal initial perturbation with parameters (47), the corresponding value of $\kappa_{l}$ is $\kappa_{c i}=0.015 \mathrm{~m}^{-1}$. For larger values of $\kappa_{l}$, the breaking distance further decreases and remains smaller than the distance to the channel origin. It formally turns to zero when $\kappa_{l}=4 / X_{0}$. Therefore, when the gradient of channel narrowing $\kappa_{l}$ is close to $4 / X_{0}$ a sinusoidal wave will break almost immediately.

So, both a narrowing of the channel and a decrease of its depth cause wave breaking, although in a different manner. It is interesting that the increase of channel width cannot completely prevent wave breaking (see line $1^{\prime}$ in Fig. 5 for the negative values of $\kappa_{l}$ ). It merely leads to smooth increasing of breaking distance. In reality, however, the governing Eq. (39) is not valid in the vicinity of the breaking point $X_{b}$ because its solutions become multi-valued (Whitham, 1974). In this case a correct description of wave process needs to take into consideration either dissipation or dispersion effects. The dispersion effects lead to creation of solitary waves and this process will be studied in the next Sect. 4. And now we shall briefly discuss the influence of dissipative effects on the breaking phenomenon.

\subsection{Influence of dissipation on wave breaking}

\subsubsection{Rayleigh dissipation}

Let us start with the simplest kind of dissipation, the Rayleigh dissipation, described by Eq. (10). The corresponding equation is

$$
\frac{\partial u}{\partial x}+\frac{1}{V(u, x)} \frac{\partial u}{\partial t}=-\frac{3}{4} \frac{v}{c_{0}(x) h^{2}(x)} u
$$

This equation also has an exact solution which can be presented in the implicit form (cf. Whitham, 1974):

$$
\begin{aligned}
u(x, t)= & \exp \left[-\frac{3}{4} v \int_{0}^{x} \frac{d \xi}{c_{0}(\xi) h^{2}(\xi)}\right] \Phi(\tau), \text { where } \\
\tau=t- & \int_{0}^{x}\left\{1-\frac{\alpha\left(x^{\prime}\right)}{s\left(x^{\prime}\right)}\right. \\
\exp & {\left.\left[-\frac{3}{4} v \int_{0}^{x^{\prime}} \frac{d \xi}{c_{0}(\xi) h^{2}(\xi)}\right] \Phi(t)\right\} \frac{d x^{\prime}}{c_{0}\left(x^{\prime}\right)} . }
\end{aligned}
$$

For the breaking distance, $X_{b}$, now we have (cf. Eq. 43):

$$
\int_{0}^{X_{b}} \frac{\alpha(x)}{s(x) c_{0}(x)} \exp \left[-\frac{3}{4} v \int_{0}^{x} \frac{d \xi}{c_{0}(\xi) h^{2}(\xi)}\right] d x=M_{d}^{-1} \text {. }
$$

In the case of variable depth channel, by substitution of $h(x)$ from Eq. (31) and simple algebra, the integral (50) can be analytically calculated to give

$u(x, t)=\exp \left\{-\frac{v}{2 \kappa_{h} h_{0}^{2} \sqrt{g h_{0}}}\left[\left(1-\kappa_{h} x\right)^{-3 / 2}-1\right]\right\} \Phi(\tau)$,

where

$$
\begin{aligned}
\tau= & t-\frac{2}{\kappa_{h} \sqrt{g h_{0}}}\left[1-\left(1-\kappa_{h} x\right)^{1 / 2}\right] \\
& +u(x, t) \exp \left[\frac{v\left(1-\kappa_{h} x\right)^{-3 / 2}}{2 \kappa_{h} h_{0}^{2} \sqrt{g h_{0}}}\right] \sqrt{\frac{2 \pi}{v \kappa_{h} \sqrt{g h_{0}}}} \\
& \times\left[\operatorname{erf}\left\{\sqrt{\frac{v}{2 \kappa_{h} h_{0}^{2} \sqrt{g h_{0}}}}\left(1-\kappa_{h} x\right)^{-3 / 4}\right\}\right. \\
& \left.-\operatorname{erf}\left\{\sqrt{\frac{v}{2 \kappa_{h} h_{0}^{2} \sqrt{g h_{0}}}}\right\}\right],
\end{aligned}
$$

and $\operatorname{erf}(x)=\frac{2}{\sqrt{\pi}} \int_{0}^{x} e^{-\xi^{2}} d \xi$ is the standard error function. Equation (51) for the breaking distance reduces to:

$$
\begin{aligned}
& \sqrt{\frac{2 \gamma_{0}(0)}{3 \kappa_{h}}}\left\{\operatorname{erf}\left[\sqrt{\frac{2 \gamma_{0}(0)}{3 \kappa_{h}}}\left(1-\kappa_{h} X_{b}\right)^{-3 / 4}\right]-\operatorname{erf} \sqrt{\frac{2 \gamma_{0}(0)}{3 \kappa_{h}}}\right\} \\
& =\frac{\gamma_{0}(0) X_{0}}{\sqrt{\pi}} \exp \left[-\frac{2 \gamma_{0}(0)}{3 \kappa_{h}}\right]
\end{aligned}
$$

where the dissipative coefficient $\gamma_{0}(x)$ is defined in Eq. (10).

Effects induced by viscosity are manifest as follows. The breaking distance increases with increasing viscosity. The critical condition when wave breaking begins impossible at negative depth gradients is modified and at small viscosities $\left(\gamma_{0}(0) X_{0} \ll 1\right)$ takes the form: $\kappa_{h} X_{0} \leq-4 / 3+4 \gamma_{0}(0) X_{0} / 9$.

For positive depth gradients, wave breaking becomes impossible if the breaking distance $X_{b}$, reaches the distance to the channel origin, $X_{o r}$, or in other words, when $\kappa_{h} X_{b}=1$. Taking into account that $\operatorname{erf}(x) \leq 1$ and using Eq. (53), this condition can be presented in the following transcendental form:

$$
\begin{gathered}
\gamma_{0}(0) X_{0}=\sqrt{\pi} \sqrt{\frac{2 \gamma_{0}(0)}{3 \kappa_{h}}} \\
\cdot\left[1-\operatorname{erf} \sqrt{\frac{2 \gamma_{0}(0)}{3 \kappa_{h}}}\right] \exp \left(\frac{2 \gamma_{0}(0)}{3 \kappa_{h}}\right) .
\end{gathered}
$$

For small viscosities, the wave breaking distance can be calculated from Eq. (53) by means of a perturbation technique resulting in: 


$$
\begin{aligned}
\frac{X_{b}}{X_{0}} \approx & \frac{1}{\kappa_{h} X_{0}}\left\{1-\left[1+\frac{3}{4} \kappa_{h} X_{0}\right.\right. \\
& \left.\left.+\frac{3}{4} \kappa_{h} X_{0}\left(1+\frac{\kappa_{h} X_{0}}{4}\right) \frac{\gamma_{0}(0) X_{0}}{2}\right]^{-4 / 3}\right\}
\end{aligned}
$$

The influence of viscosity on wave breaking in a channel of linearly varying depth is illustrated in Fig. 5 (compare lines 1 and 2 calculated at $\left.\gamma_{0}(0) X_{0}=0.3\right)$. As one can see, the correction caused by viscosity leads to the increase of the breaking distance although the general dependence of $X_{b}$ on $\kappa_{h}$ remains the same as in the inviscid case.

In the case of a channel of variable width, by substitution of $l(x)$ from Eq. (35) and simple algebra, the condition determining the breaking distance (51) can be presented as

$$
\int_{0}^{\kappa_{l} X_{b}} \exp \left(-\frac{3}{4} \frac{\nu \xi}{\kappa_{l} c_{0} h^{2}}\right) \frac{d \xi}{\sqrt{1-\xi}}=\frac{2}{3} \frac{c_{0} \kappa_{l} h}{M_{d}} \equiv \kappa_{l} X_{0} .
$$

Unfortunately, this integral cannot be expressed through elementary functions. However, if one assumes that $\left|\kappa_{l} X_{b}\right| \ll 1$ (this can be checked afterwards), the integral in Eq. (56) can be calculated approximately by decomposing the integrand into a Taylor series to yield

$$
\begin{aligned}
& \int_{0}^{\kappa_{l} X_{b}}\left[1+\left(1-\frac{2 \gamma_{0}}{\kappa_{l}}\right) \frac{\xi}{2}\right] d \xi \\
& \quad \equiv \kappa_{l} X_{b}+\left(1-\frac{2 \gamma_{0}}{\kappa_{l}}\right) \frac{\left(\kappa_{l} X_{b}\right)^{2}}{4} \approx \kappa_{l} X_{0} .
\end{aligned}
$$

The solution of this equation is

$$
\frac{X_{b}}{X_{0}} \approx \frac{2}{\kappa_{l} X_{0}} \frac{\sqrt{1+\kappa_{l} X_{0}\left(1-2 \gamma_{0} / \kappa_{l}\right)}-1}{1--2 \gamma_{0} / \kappa_{l}} .
$$

In Fig. 5 the solution (58) is depicted by the dashed line $2^{\prime}$ for $\gamma_{0} / \kappa_{l}=0.1$. As one can see, viscosity leads to an increase of the breaking distance which can then become formally larger than $X_{o r}$ so that breaking becomes impossible. This corresponds in Fig. 5 to the intersection of curves $2^{\prime}$ and 3.

The range of validity of Eq. (58) is restricted by the condition $\left|\kappa_{l} X_{b}\right| \ll 1$ (see above) which results in $\left|\kappa_{l} X_{0}\right| \ll 1$.

\subsubsection{Chezy dissipation}

The main governing equation in this case is

$\frac{\partial u}{\partial x}+\frac{1}{V(u, x)} \frac{\partial u}{\partial t}=-\frac{k}{h^{2}(x) s(x)}|u| u$.

This equation can also be solved analytically and the exact solution presented in the following implicit form:

$$
u(x, t)=\Phi(t)\left[1+k|\Phi(t)| \int_{0}^{x} \frac{d \xi}{h^{2}(\xi) s(\xi)}\right]^{-1}
$$

The breaking distance, $X_{b}$, at which the discontinuity appears, is such that (cf. Eq. 43):

$$
\int_{0}^{X_{b}}\left[1+k|\Phi(t)| \int_{0}^{x} \frac{d \xi}{h^{2}(\xi) s(\xi)}\right]^{-2} \frac{\alpha(x) d x}{s(x) c_{0}(x)}=M_{d}^{-1} \text {. }
$$

This transcendental equation is too complex to be analyzed in general. However, in the case of a channel of linearly tapered width, a solution of this equation can be found explicitly. Using again Eq. (35) after simple manipulations one obtains

$$
\begin{aligned}
\frac{X_{b}}{X_{0}} & =\frac{1}{1-k \min [|\Phi|] X_{0} / h^{2}} \\
& \cdot\left(1-\frac{\kappa_{l} X_{0}}{1-k \min [|\Phi|] X_{0} / h^{2}}\right)=1-\kappa_{l} X_{0},
\end{aligned}
$$

where the natural condition $\min [|\Phi(t)|]=0$ is applied.

Several interesting observations follow from this solution. As was pointed out in Pelinovsky (1996), in the homogeneous case Chezy dissipation cannot prevent wave breaking which occurs at the point where $u(x, t)=0$. Moreover, breaking distance in the viscous case $X_{b}$ is exactly the same as in the inviscid one, $X_{0}$.

\subsubsection{Reynolds dissipation}

The governing equation in this case is a generalized Burgers equation Whitham (1974):

$\frac{\partial u}{\partial x}+\frac{1}{V(u, x)} \frac{\partial u}{\partial t}=\frac{v}{c_{0}^{3}(x)} \frac{\partial^{2} u}{\partial t^{2}}$.

Exact solutions of this inhomogeneous equation are not known in general. Nevertheless, some estimations and qualitative description of wave evolution can be made by approximating the equation by a pure Burgers equation with constant coefficients

$\frac{\partial u}{\partial x}+\frac{1}{c_{0}}\left(1-\frac{3}{2} \frac{u}{h_{0}}\right) \frac{\partial u}{\partial t}=\frac{v}{c_{0}^{3}} \frac{\partial^{2} u}{\partial t^{2}}$,

that is completely integrable by means of the Hopf-Cole transformation and its solutions are very well studied (see, e.g. Whitham, 1974). We give its most important properties in connection with river wave dynamics.

- The equation does not give rise to wave breaking at all. In the process of evolution, an initial perturbation steepens until it reaches some extreme value which depends on the dissipation parameter $v$. The smaller the parameter $v$, the thinner the wave front.

- A sinusoidal initial perturbation evolves into a sawtooth wave with a thin frontal part and a long almost linear rear part.

- The dissipation takes place only on the frontal part of a saw-tooth wave where the wave profile differs from the linear function (the second derivative in the right-hand side of Eq. (64) responsible for the dissipation, is zero for linear functions). 
- The equation possesses stationary solutions in the form of shock waves which can be considered as models of tidal bores (mascaret) moving along the river from its mouth.

To conclude this section on the role of dissipation on wave breaking, its influence depends on the model used. Rayleigh dissipation increases the breaking distance of Riemann waves and eliminates breaking if the channel depth or width decrease too fast. Chezy dissipation does not impact on wave breaking at all, while Reynolds dissipation always prevents wave breaking and gives rise to either triangle (sawtooth) waves or step-wise shock waves. In the next section we consider the influence of dispersion on steep waves in the case when Eq. (8) cannot be reduced to the simple wave equation (39). As a model we consider a step-wise initial profile which can be generated by a tide. Dispersion leads to the disintegration of such a step-wise perturbation into stable particle-like nonlinear waves - solitons. This process is very well-known (see, e.g. Whitham, 1974), and we study it in the particular case of a channel of decreasing depth or width. We also study the influence of different types of dissipation on the disintegration of a step-wise perturbation and on the emergence of solitons.

\section{Bore disintegration into KdV solitons}

Rivers which flow to the open oceans are usually affected by tidal effects. Tidal motions generate intensive water flows which can propagate upstream on tens of kilometers in the form of step-wise perturbations (hydraulic jumps) analogous to shock waves in acoustics. This phenomenon is known as a bore or a mascaret in French. In the process of propagation the bore undergoes dissipation, dispersive disintegration, enhancement due to decrease of the river width and depth, influence of nonlinear effects, etc. We study these effects here both theoretically and by means of numerical modelling.

First of all, consider bore disintegration into solitons in a channel of constant parameters within the framework of $\mathrm{KdV}$ equation. This equation follows from Eq. (24) if one omits energy dissipation $(F(\eta) \equiv 0)$. The function $s(x) \equiv 1$ in this case (see Eq. 23) and the resultant equation is:

$\frac{\partial \eta}{\partial x}+\frac{1}{c_{0}}(1-\alpha \eta) \frac{\partial \eta}{\partial t}-\beta \frac{\partial^{3} \eta}{\partial t^{3}}=0$,

where coefficients $c_{0}, \alpha, \beta$ are given by Eq. (9) with constant value of $h$.

Let us suppose that the bore can be represented by a Heaviside function of amplitude $U$. Making the transformation

$v=\frac{\eta}{U} ; \quad \xi=x \sqrt{\frac{\alpha^{3} U^{3}}{\beta c_{0}^{3}}} ; \quad \tau=\sqrt{\frac{\alpha U}{\beta c_{0}}}\left(t-\frac{x}{c_{0}}\right)$,

one can reduce this equation to the dimensionless form:

$$
\frac{\partial v}{\partial \xi}-v \frac{\partial v}{\partial \tau}-\frac{\partial^{3} v}{\partial \tau^{3}}=0
$$

The boundary condition for the function $v$ is just the unit Heaviside function in this case: $v(0, \tau)=H(\tau)$. As the boundary perturbation, i.e. the Heaviside function, does not contain any temporal parameter, the Cauchy problem for Eq. (67) does not contain any free parameters. So we can describe the disintegration of a step-wise function into solitons in the dimensional variables by solving the problem for Eq. (67) once in the dimensionless variables. Then one can return to the dimensional variables for each different set of parameters and in particluar, for each different initial amplitude $U$.

An interesting practical problem is the determination of the distance when the first soliton emerges from the "initial" step-wise perturbation. This distance cannot be determined exactly because the soliton emerges gradually and, formally speaking, it completely appears only at infinity. But one can suggest different practical criterions when the first pulse can be considered as a KdV soliton with a good accuracy, e.g. when its amplitude amounts $90 \%$ of the theoretical value or when the local minimum of the wave field behind it amounts to $10 \%$ of its maximum. This last criterion has been used by one of the authors in Pelinovsky and Stepanyants (1981) to study experimentally the process of disintegration of a stepwise perturbation into solitons using an electromagnetic LCtransmission line. In any case, if the dimensionless distance $\xi_{s}$ of the first soliton emergence has been determined somehow, then in the original variables $u, x, t$ the dimensional distance $X_{s}$ can be expressed using the relation between $x$ and $\xi$ in Eq. (66):

$$
X_{s}=\xi_{s} \sqrt{\frac{\beta c_{0}^{3}}{\alpha^{3} U^{3}}} .
$$

The problem of disintegration of a step-wise perturbation in a homogeneous medium was studied by different authors Gurevich and Pitaevskii (1974); Khruslov (1975); Merchant and Smyth (1991); Pelinovsky and Stepanyants (1981). It was confirmed that this process is self-similar. The perturbation evolves into an infinite sequence of solitons whose amplitudes eventually amount to double the amplitude of the initial perturbation and the time delay between them growths logarithmically with distance Khruslov (1975). Essentially the same problem has been studied for surface gravity waves in Merchant and Smyth (1991) by means of the initial-boundary value problem for the original KdV Eq. (1), rather than for its signalling version Eq. (67). Note that the same problem of bore disintegration for surface capillary waves uses a slightly different technique within the framework of the initial-boundary value problem Merchant and Smyth (2002).

The dimensionless distance when the first soliton emerges according to the criterion suggested in Pelinovsky and Stepanyants (1981) has been estimated using numerical calculations and data from the transmission line mentioned above to be $\xi_{s} \approx 45$. So, the dimensional distance $X_{s}$ can be presented in our case as a function of channel depth $h$ and amplitude of 
Table 3. The dispersion coefficient $B$ for different variations of the depth or width of the channel

\begin{tabular}{lcc}
\hline \multicolumn{1}{c}{ Variable } & Depth & Width \\
\hline Linear & $B_{d l}(\xi)=\left(1+\frac{3}{4} \frac{\xi}{\xi}\right)^{-3}$ & $B_{w l}(\xi)=1-\frac{1}{2} \frac{\xi}{\xi}$ \\
\hline Exponential & $B_{d e}(\xi)=\left(1+\frac{7}{4} \frac{\xi}{\xi}\right)^{-9 / 7}$ & $B_{w e}(\xi)=\left(1+\frac{1}{2} \frac{\xi}{\xi}\right)^{-1}$ \\
\hline
\end{tabular}

initial perturbation $U$ :

$X_{s}=45 U^{-3 / 2} \sqrt{\frac{\beta c_{0}^{3}}{\alpha^{3}}}=10 h^{5 / 2} U^{-3 / 2}$.

By substitution $U=1 \mathrm{~m}, h=10 \mathrm{~m}$, one obtains $X_{s} \approx$ $3160 \mathrm{~m}$ while for the same depth and $U=2 \mathrm{~m}$ this distance is about three times shorter, $X_{s} \approx 1120 \mathrm{~m}$. It changes much more when the channel depth varies, for example, $U=1 \mathrm{~m}$ and $h=5 \mathrm{~m}$, give $X_{s} \approx 559 \mathrm{~m}$. Note also that we have verified directly from numerical simulations the dependence of $X_{s}$ on $\beta$ given by Eq. (69) and the agreement is very good.

In inhomogeneous media the determination of $X_{S}$ is more complex because the problem is not self-similar but depends on the characteristic scale of inhomogeneity. Unfortunately, there are no analytical solutions in this case in general. However, if the inhomogeneity is very smooth so that its characteristic length is much greater than $X_{s}$, then Eq. (69) still can be used as a rough estimation of the first soliton emergence.

In the general case of an inhomogeneous channel the following approach to the problem of bore disintegration can be developed. Let us start again from Eq. (24) omitting energy dissipation $(F(u) \equiv 0)$ but bearing in mind that the original variable $\eta(x, t)$ is now linked to the auxiliary variable $u(x, t)$ by Eq. (23). Assume again that the boundary perturbation is the Heaviside function of the amplitude $U$ and make the transformation:

$$
\begin{aligned}
& v=\frac{u}{U} ; \quad \xi=U^{3 / 2} \sqrt{\frac{\alpha(0)}{\beta(0) c_{0}(0)}} \int_{0}^{x} \frac{\alpha\left(x^{\prime}\right) d x^{\prime}}{c_{0}\left(x^{\prime}\right) s\left(x^{\prime}\right)} \\
& \tau=\sqrt{\frac{U \alpha(0)}{\beta(0) c_{0}(0)}}\left(t-\int_{0}^{x} \frac{d x^{\prime}}{c_{0}\left(x^{\prime}\right)}\right)
\end{aligned}
$$

In the new variables the $\mathrm{KdV}$ equation takes the form

$$
\frac{\partial v}{\partial \xi}-v \frac{\partial v}{\partial \tau}-B(\xi) \frac{\partial^{3} v}{\partial \tau^{3}}=0
$$

where

$B(\xi)=s(\xi)\left[\frac{h(\xi)}{h(0)}\right]^{2}$

with the boundary condition $v(0, \tau)=H(\tau)$.

We consider two main situations, a linear or exponential decrease of the depth $h$ (resp. the width $w$ ) of the channel in the form $h=h_{0}\left(1-\kappa_{h} x\right)$ or $h=h_{0} e^{-\kappa_{h} x}$ (resp.

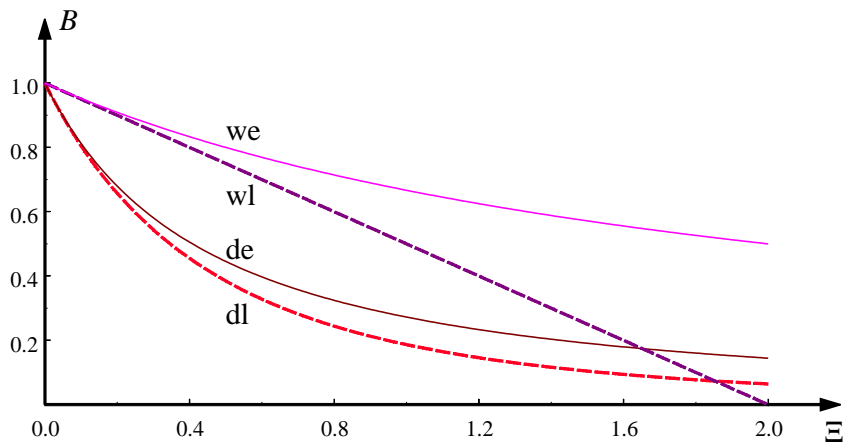

Fig. 6. The dependence of the dispersion parameter $B$ of Eq. (71) and Table 3 for channels of linearly decreasing depth $(d l)$, exponentially decreasing depth $(d e)$ linearly decreasing width $(w l)$ and exponentially decreasing width (we).

$l=l_{0}\left(1-\kappa_{l} x\right)$ and $\left.l=l_{0} e^{-\kappa_{l} x}\right)$. The two types of variation present different features and a linear decay can be considered as a first approximation to the exponential decay. Note also that according to Mazumber and Bose (1995), "tidal rivers generally taper at an exponential rate", and data for Hugli River, India agree with this statement very well, providing $l_{0}=21.5 \mathrm{~km}, \kappa_{l}=2.7 \cdot 10^{-5} \mathrm{~m}^{-1}$.

We present in Table 3 the expressions for the dispersion coefficient $B$ obtained for the different types of variation. All these expressions can be cast in the form $B(\Xi)$ where $\Xi=$ $\xi / \bar{\xi}$ where the normalized $\bar{\xi}$ is

$\bar{\xi}=\frac{9}{2} \frac{U^{3 / 2}}{\kappa h_{0}^{5 / 2}}$,

where $\kappa$ is the corresponding gradient of depth or width variations.

The behaviour of $B$ from Table 3 is displayed in Fig. 6 as a function of the normalized variable $\Xi$. In all the cases presented $B$ decreases as one moves upstream going to larger $\xi$ so that nonlinearity will increasingly dominate dispersion. This will cause solitons to form earlier than in the homogeneous case so that the distance of first soliton emerging will be smaller than the one given by Eq. (69) which can be considered as an upper bound.

One can see that $B(\Xi)$ decreases faster when the depth of the channel is decreased rather than the width so that in the former situation nonlinear effects are reinforced leading for this case to an even smaller distance of first soliton emerging. We observe the same effect as for the breaking distance of Riemann waves discussed in Sect. 3. Note also that a linear decay of the width gives rise to a pathological vanishing of $B$ for $\xi=2 \bar{\xi}$, this feature is absent in the case of an exponential taper of the river width.

The influence of dissipation on this decomposition process has not been studied so far, however it is very well-known that Reynolds dissipation can result in the formation of a bore with a stationary front, either oscillatory or monotonic (Whitham, 1974). We study the process of bore disintegra- 
tion into solitons numerically in Sect. 7 both without dissipation and with different types of dissipation.

\section{Soliton creation from small perturbations}

Let us assume now that the initial perturbation has a small amplitude $U$ or duration $T$ so that the parameter $\sigma^{2}$ defined in Eq. (29) is much smaller than 12 - the soliton generic value (Ablowitz and Segur, 1981; Pelinovsky and Stepanyants, 1981; Whitham, 1974). When $\sigma^{2} \ll 12$ the dispersion term dominates the nonlinear one in Eq. (29) and, hence, the nonlinear term can be omitted (we also neglect the dissipation effects). As a result, Eq. (29) reduces to the variable coefficient linearized $\mathrm{KdV}$ equation:

$\frac{\partial v}{\partial \zeta}-\frac{1}{\sigma^{2}(\zeta)} \frac{\partial^{3} v}{\partial \theta^{3}}=0$

By means of the transformation

$\chi=\int_{0}^{\zeta} \frac{d \zeta^{\prime}}{\sigma^{2}\left(\zeta^{\prime}\right)}, \quad \vartheta=-\theta$

the equation can be further reduced to the constant coefficients one:

$\frac{\partial v}{\partial \chi}+\frac{\partial^{3} v}{\partial \vartheta^{3}}=0$

The asymptotic solution of this equation is very wellknown and can be expressed through the Airy function (Karpman, 1973):

$v(\chi, \theta)=\frac{1}{\sqrt{\pi}(3 \chi)^{1 / 3}} \operatorname{Ai}\left[\frac{\vartheta}{(3 \chi)^{1 / 3}}\right]$.

Note, that this solution is universal and does not depend on the detailed form of the initial perturbation. In the variables $\zeta, \theta$ the solution is

$$
\begin{gathered}
v(\zeta, \theta)=\frac{1}{\sqrt{\pi}}\left[3 \int_{0}^{\zeta} \frac{d \zeta^{\prime}}{\sigma^{2}\left(\zeta^{\prime}\right)}\right]^{-1 / 3} \\
\cdot \operatorname{Ai}\left\{-\theta\left[3 \int_{0}^{\zeta} \frac{d \zeta^{\prime}}{\sigma^{2}\left(\zeta^{\prime}\right)}\right]^{-1 / 3}\right\} .
\end{gathered}
$$

According to this solution, the amplitude and duration of the perturbation vary as

$$
\begin{aligned}
& U(\zeta) \sim\left[\int_{0}^{\zeta} d \zeta^{\prime} / \sigma^{2}\left(\zeta^{\prime}\right)\right]^{-1 / 3}, \\
& T(\zeta) \sim\left[\int_{0}^{\zeta} d \zeta^{\prime} / \sigma^{2}\left(\zeta^{\prime}\right)\right]^{1 / 3},
\end{aligned}
$$

so that the total wave mass is conserved during the wave evolution, $M \sim U(\zeta) T(\zeta)=$ const. Considering the solution (77) as an "initial" perturbation at each spatial point, one can define a "current" value of the Ursell parameter $\sigma_{\text {cur }}^{2}(\zeta) \sim U(\zeta) T^{2}(\zeta)$ (cf. with the formula after Eq. 29). This parameter does not remain constant in general but varies in space. If it growths, the perturbation becomes larger so that the nonlinear term $\sim v \frac{\partial v}{\partial \theta}$ in Eq. (29) cannot be neglected anymore and must be taken into account. From a practical point of view, the front part of the perturbation evolves into a $\mathrm{KdV}$ soliton in this case. The distance for which the "current" Ursell parameter reaches its "soliton" value, $\sigma_{s}^{2}=12$, can be taken as the distance $\zeta_{s}$ of soliton creation from a small initial perturbation. This distance can be estimated from the following equation (Pelinovsky and Stepanyants, 1981):

$$
\begin{aligned}
\sigma_{c u r}^{2}\left(\zeta_{s}\right) & \equiv \frac{p(\zeta)}{\beta(\zeta)} U(\zeta) T^{2}(\zeta) \\
& =\frac{p\left(\zeta_{s}\right)}{\beta\left(\zeta_{s}\right)} \frac{\left(U_{0} T_{0}^{2}\right)^{2 / 3}}{\sqrt{\pi}}\left[3 \int_{0}^{\zeta_{s}} \frac{\beta\left(\zeta^{\prime}\right) d \zeta^{\prime}}{p\left(\zeta^{\prime}\right)}\right]^{1 / 3}=12,
\end{aligned}
$$

where $U_{0}$ and $T_{0}$ are respectively the initial amplitude and duration of the given perturbation.

Further development can be done if the channel inhomogeneity is specified. Suppose again that the channel depth decreases linearly with distance. Substituting the expressions for $p(\zeta)$ and $\beta(\zeta)$ and after some algebra one can obtain a transcendental equation for the distance $X_{e}$ at which a soliton emerges:

$$
\frac{\left[1-\left(1-\kappa_{h} X_{e}\right)^{3 / 2}\right]^{1 / 3}}{\left(1-\kappa_{h} X_{e}\right)^{9 / 4}}=\frac{4 \sqrt{\pi}}{9^{1 / 3} g^{1 / 2}} \frac{\kappa_{h}^{1 / 3} h_{0}^{11 / 6}}{U_{0} T_{0}} .
$$

By substitution into this equation of the following parameters:

$h_{0}=10 \mathrm{~m}, \quad \kappa_{h}=10^{-4} \mathrm{~m}^{-1}, \quad U_{0}=1 \mathrm{~m}, \quad T_{0}=1 \mathrm{~s}$,

corresponding to $\sigma^{2} \approx 1 \ll 12$, one obtains $X_{e}=4637 \mathrm{~m}$. The channel depth at this distance is $h=5.4 \mathrm{~m}$

If the inhomogeneity is a linear decrease of the channel width, then the distance of soliton emerging can be estimated from the equation:

$$
\frac{\left(\kappa_{l} X_{e}\right)^{1 / 3}}{\left(1-\kappa_{l} X_{e}\right)^{1 / 4}}=\frac{2^{7 / 3} \sqrt{\pi}}{3} \frac{\kappa_{l}^{1 / 3} h^{7 / 3}}{c_{0} U_{0} T_{0}} .
$$

For the same parameters as above, assuming $h=10 \mathrm{~m}$, $\kappa_{l}=10^{-4} \mathrm{~m}^{-1}$, it follows from Eq. (81) $X_{e}=9880 \mathrm{~m}$. At this distance the channel width is $l=12 \mathrm{~m}$ while initially it was $l_{0}=1000 \mathrm{~m}$.

Note in addition that for some dependences of the parameters $p(\zeta)$ and $\beta(\zeta)$, the Ursell parameter can remain constant or even decrease with $\zeta$. This means that a small-amplitude initial perturbation will not evolve into a soliton, and can be completely described within the framework of the linear 
theory. This is the case in particular, if the channel depth increases (instead of decreasing) faster than $h(\zeta) \sim \zeta^{1 / 2}$ and the width remains constant. In the dimensional variable the critical dependence of a channel depth on distance is $h(x) \sim x^{4 / 15}$.

\section{Adiabatic decay of KdV solitons}

Let us assume that the initial perturbation is a $\mathrm{KdV}$ soliton and calculate its decay in an homogeneous channel due to the different types of dissipation listed in Sect. 2.2. We therefore consider Eq. (8) with constant coefficients omitting the last term in the left-hand side since $\Delta$ is constant. We also assume the dissipation to be small enough so that the approximate approach of Sect. 2.3 based on conservation laws can be applied again. As a trial solution of Eq. (8) we consider a KdV soliton described by Eq. (20). This solution is exact if the dissipation is absent $(F(\eta)=0)$. In the spirit of the adiabatic approach, it is assumed that for small dissipation the structure of the soliton is preserved unchanged but that its parameters vary in space. The variation of the soliton amplitude can be obtained from the equation of energy balance.

Consider first Rayleigh dissipation described by Eq. (10) for the function $F(\eta)$. Multiplying Eq. (8) by $\eta$ and integrating it over $t$ from minus to plus infinity, one obtains

$\frac{d<\eta^{2}>}{d x}=-2 \gamma_{0}<\eta^{2}>$,

where

$<\eta^{2}>=\int_{-\infty}^{+\infty} \eta^{2}(x, t) d t$.

By substitution of the soliton solution, Eq. (20), into this equation and taking into account the relationship between the soliton amplitude and duration, one obtains

$\frac{d A}{d x}=-\frac{4}{3} \gamma_{0} A$.

The solution of this equation leads to the very well-known result (see, e.g. Ott and Sudan, 1970; Pelinovsky, 1971): soliton amplitude decays exponentially in space with an exponent of decay $4 / 3$ times greater than in the linear case

$A(x)=A_{0} \exp \left[-\frac{4}{3} \gamma_{0}\left(x-x_{0}\right)\right]$.

In a similar way the decay of the soliton amplitude can be found for Chezy, Reynolds and other types of dissipations (cf., for instance, Kakutani and Matsuuchi (1975); Ott and Sudan (1970); Pelinovsky (1971)). We summarize the results in Table 4 using expressions for the decay coefficients $\gamma_{0}, \gamma_{c}$ and $\gamma_{2}$ (see Eqs. 10 to 12). We also include into the Table the decay law for the integral dissipation obtained in Kakutani and Matsuuchi (1975); Miles (1976).

In the case of integral dissipation (the last row of the table), $v$ stands for the kinematic viscosity of water, while in the cases of Rayleigh and Reynolds dissipation, $v$ must be replaced by some effective turbulent viscosity as mentioned in Sect. 2.2.

In all cases except Rayleigh dissipation, the soliton amplitude for large distances does not depend on the initial value $A_{0}$ and decays algebraically. The decay law for Chezy and Reynolds dissipation is proportional to $x^{-1}$, while for the integral dissipation it is proportional to $x^{-4}$. In this last case the soliton amplitude for large distances strongly depends on the channel depth, $A \sim h^{8}$, while for Chezy dissipation $A \sim h^{2}$ and for Reynolds dissipation $A \sim h^{7 / 2}$.

The effect of dissipation on nonlinear wave trains was described in Myint and Grimshaw (1995) using modulation theory. Unfortunately, the set of equations obtained for the modulation parameters of the nonlinear wave are too complex to be analyzed even in a uniform medium. This is why we restricted ourselves to the case of a single soliton.

The combination of dissipation and inhomogeneity results in an increase or a decrease of the soliton amplitude with distance. For some special conditions, these two effects can compensate each other and soliton amplitude can remain constant. To illustrate this we consider here the simplest case of Rayleigh dissipation in an inhomogeneous medium. Applying again the energy balance equation, one obtains from Eq. (8)

$\frac{1}{2} \frac{d}{d x}<\eta^{2}>+\frac{1}{2} \frac{<\eta^{2}>}{\Delta} \frac{d \Delta}{d x}=-\gamma_{0}(x)<\eta^{2}>$.

The solution of this equation can be presented in a closed form

$<\eta^{2}>\Delta(x)=<\eta_{0}^{2}>\Delta_{0} \exp \left(-2 \int_{x_{0}}^{x} \gamma_{0}\left(x^{\prime}\right) d x^{\prime}\right)$,

where $\eta_{0}$ and $\Delta_{0}$ stand for that the corresponding variables at $x=x_{0}$.

By substituting in Eq. (86) the soliton solution Eq. (20) and using Eqs. (9), (10) for the parameters $\alpha, \beta, \gamma_{0}$ and $\Delta$, one obtains

$A(x)=A_{0} \frac{h_{0}}{h(x)}\left(\frac{l_{0}}{l(x)}\right)^{2 / 3} \exp \left(-\int_{x_{0}}^{x} \frac{v d x^{\prime}}{c_{0}\left(x^{\prime}\right) h^{2}\left(x^{\prime}\right)}\right)$.

This formula generalizes Green's law, Eq. (22), and Rayleigh dissipation law Eq. (84) for a KdV soliton. Assuming $v=$ const, one readily obtains from Eq. (87) for a channel of variable depth but constant width that the soliton amplitude remains unchanged, $A=A_{0}$, if

$h(x)=h_{0}\left[1-\frac{5}{2} \frac{v}{\sqrt{g h_{0}^{5 / 2}}}\left(x-x_{0}\right)\right]^{2 / 5}$.

If the depth decreases faster than described by this formula, soliton amplitude will increase, otherwise it will decrease. Similar conditions can be derived for other types of dissipation. 
Table 4. Dissipation laws for soliton amplitude for different types of dissipation

\begin{tabular}{ll}
\hline Type of dissipation & Soliton amplitude decay \\
\hline Rayleigh & $A(x)=A_{0} \exp \left[-\frac{4}{3} \gamma_{0}\left(x-x_{0}\right)\right]=A_{0} \exp \left[-\frac{v}{\sqrt{g} h^{5 / 2}}\left(x-x_{0}\right)\right]$ \\
Chezy & $A(x)=\frac{A_{0}}{1+\frac{16}{15} \gamma_{c} A_{0}\left(x-x_{0}\right)}=\frac{A_{0}}{1+\frac{16}{15} \frac{k}{h^{2}} A_{0}\left(x-x_{0}\right)}$ \\
\hline Reynolds & $A(x)=\frac{A_{0}}{1+\frac{2}{5} \frac{g}{h^{2}} \gamma_{2} A_{0}\left(x-x_{0}\right)}=\frac{A_{0}}{1+\frac{2}{5} \frac{v}{\sqrt{g} h^{7 / 2}} A_{0}\left(x-x_{0}\right)}$ \\
\hline Integral dissipation & $A(x)=\frac{A_{0}}{\left[1+0.1677(1+h / l) \sqrt[4]{\frac{A_{0} v^{2}}{g}} h^{-2}\left(x-x_{0}\right)\right]^{4}}$
\end{tabular}

\section{Numerical modelling}

We studied numerically the propagation of long surface waves in a nonuniform channel using the dimensionless Eq. (29). An explicit central-difference scheme was used (the details are given in Pelinovsky et al., 1993). This scheme is second-order accurate both in space and time and is conditionally stable provided $\Delta x \sim \Delta t^{3}$, where $\Delta x$ and $\Delta t$ are the spatial and temporal steps. The simple models of dissipation (10)-(12) were taken into account. The modification of this conservative finite-difference scheme for $\mathrm{KdV}$ equation to account for the dissipative terms (10) or (11) is straightforward. For the Burgers type dissipation described by Eq. (12), the Duffort-Frankel discretization was used (MacKraken and Dorn, 1975). It keeps the scheme accuracy and explicitness. Assuming no dissipation we first describe soliton formation in a channel of linearly decreasing depth and compare the situations of exponentially decreasing depth and width. After that we show the influence of dissipation in the particular example of a channel of linearly decreasing depth.

We assume that the initial perturbation is the step-wise function:

$\eta(0, t)=\frac{U_{0}}{2}\left(1+\tanh \frac{t-t_{0}}{T_{0}}\right)$,

where $U_{0}$ is the amplitude, $t_{0}$ is the conditional instant of time when the front of the perturbation arrives at the point of observation and $T_{0}$ is the characteristic front duration. (In fact we used a combination of such step-wise functions with amplitudes of opposite signs and shifted fronts so that the initial perturbation was pulse-like with very long duration, so that the rear slope of the pulse did not affect the wave processes occurring in the frontal zone.)

Throughout the section we choose the following values for the parameters: $h_{0}=10 \mathrm{~m}$ and $U_{0}=1 \mathrm{~m}$.

In a first experiment we consider a channel with a linear depth gradient $\kappa_{h}=10^{-3} \mathrm{~m}^{-1}$. Time dependence of the solution to the KdV Eq. (29) at different locations are shown in Fig. 7. Specifically on the top panel we present the solution $v(\xi, \tau)$ in the reduced variables for $\xi=0, \xi=18$ and $\xi=30$. Notice how the front part of the bore gives rise to solitons. The leading pulse is well formed despite $\xi=30<45$ and its amplitude is larger than 2 due to the inhomogeneity effect $(B(\xi)<1)$. The bottom panel of Fig. 7 shows the solution for water surface perturbation $\eta(x, t)$ in the laboratory frame for the two last locations $x=532$ $(\xi=18)$ and $x=644(\xi=30)$. Notice the main features of the transformations (23) and (25), the amplification of the wave due to the inhomogeneity and the time shift due to Galilean transformation.

We now compare the two types of channel inhomogeneity for the same gradient $\kappa=10^{-3} \mathrm{~m}^{-1}$. We chose an exponential decrease of the width or depth. From Table 3 and Fig. 6 we know that a decrease of the channel depth will give rise to a smaller dispersion parameter $B(\xi)$ than when the width is decreased. We then expect a smaller distance of emergence of the first soliton. Figure 8 shows the plot of water surface perturbation $\eta(x, t)$ for $x=10^{3} \mathrm{~m}$ for a channel of exponentially decreasing depth and exponentially decreasing width. As expected, narrow and high solitons are well formed in the first case while they are just emerging from the initial step-wise perturbation in the second case. Once soli- 


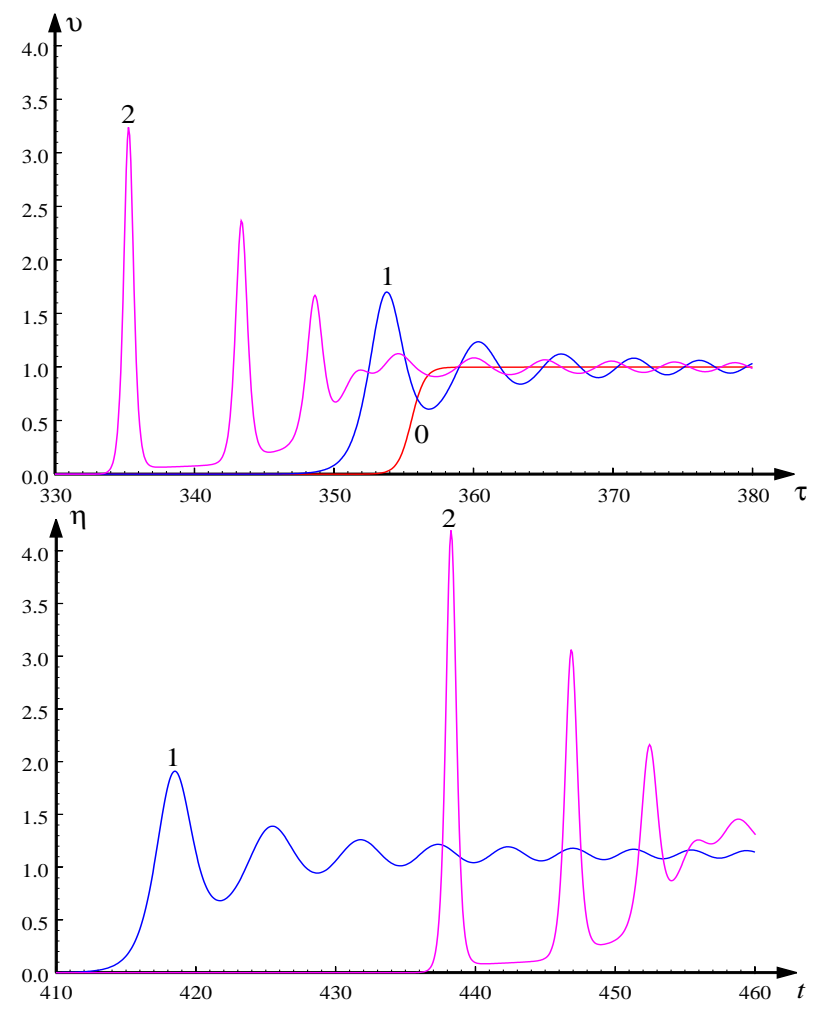

Fig. 7. Bore decomposition into solitons for a channel of linearly decreasing depth. The top panel shows time dependence of the solution $v(\xi, \tau)$ in the reduced coordinates for the initial condition $\xi=0$ (curve 0 ), $\xi=18$ (curve 1 ) and $\xi=30$ (curve 2 ). The bottom panel shows the corresponding perturbation of water surface in the laboratory frame $\eta(x, t)$ for the last two values of $\xi, x=532 \mathrm{~m}$ $(\xi=18)$ and $x=644 \mathrm{~m}(\xi=30)$.

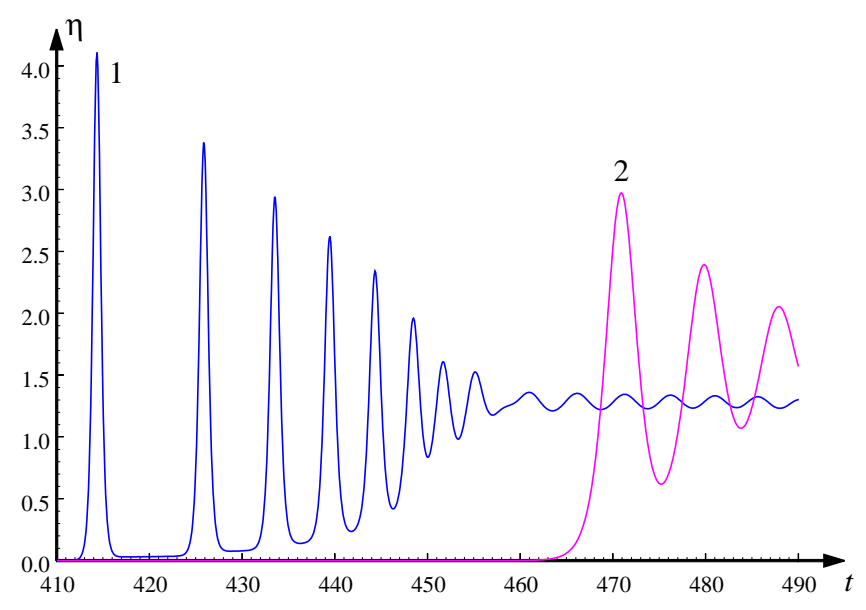

Fig. 8. Dependence of water surface perturbation $\eta(x, t)$ on time at $x=10^{3} \mathrm{~m}$ for a channel of exponentially decreasing depth (curve 1) and exponentially decreasing width (curve 2).

tons are created they evolve practically independently from each other and their amplitudes varies according to the nonlinear Green's law $A \sim h^{-1}(x) l^{-2 / 3}(x)$ (see Eq. 22 in the Sect. 2.3). This is clearly seen on of Fig. 9 where the leading

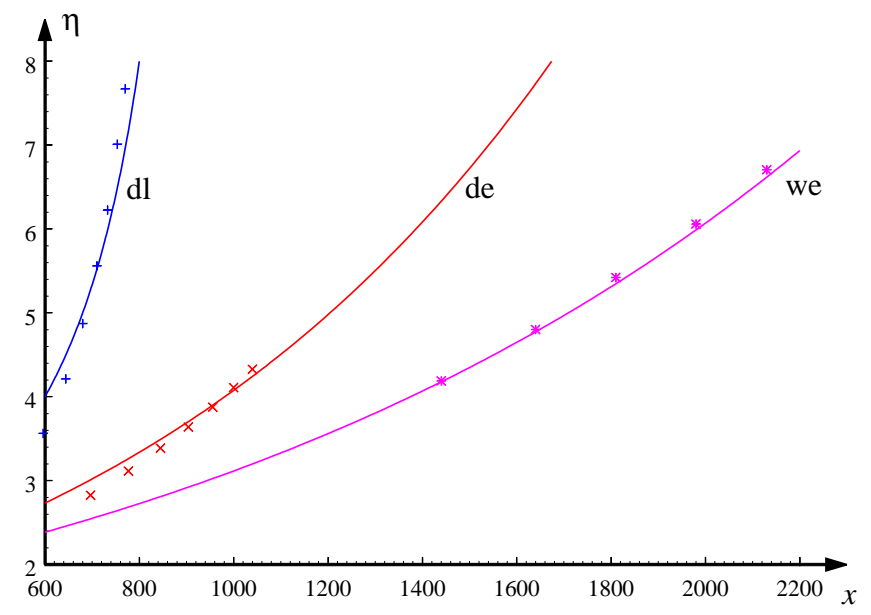

Fig. 9. The leading pulse amplitude shown in Fig. 8 as a function of $x$ computed numerically (symbols) for a channel of linearly decreasing depth $(d l)$, exponentially decreasing depth $(d e)$ and exponentially decreasing width (we). The corresponding Green's laws (22) are given for each case in solid lines.

pulse amplitude has been plotted for a linear decrease of the depth $(d l)$, an exponential decrease of the depth $(d e)$ and an exponential decrease of the width (we).

We now consider the influence of dissipation in the particular case of a channel of linearly decreasing depth $\kappa_{h}=$ $10^{-4} \mathrm{~m}^{-1}$. Fig. 10 shows time dependence of the watersurface perturbation $\eta(x, t)$ for $x=1080 \mathrm{~m}$ (top panel) and $x=2930 \mathrm{~m}$ (bottom panel). The inviscid solution is shown in solid line while the one obtained for a Chezy damping of $k=10^{-3}$ is presented in broken line. Note how the amplitudes of the solitons are strongly reduced by the damping. As the soliton velocities are related to their amplitudes (see the relationships after Eq. 20), there are temporal phase shifts between the corresponding solitons in the viscid and inviscid cases. These shifts increase gradually with distance as can be seen in Fig. 10.

The first soliton, once it is formed, evolves almost independently from the other solitons. Its amplitude increases in accordance with the nonlinear Green's law, $A \sim h^{-1}(x)$ (see Sect. 2.3). This dependence has been confirmed in the numerical modelling and is depicted in Fig. 11 (compare the solid line 1 and the dashed line asymptotically approaching the solid one). In the first stage of the bore evolution, the amplitude of the perturbation grows mainly due to the formation of a soliton. According to the theory for a uniform channel, the first soliton at the front is the biggest and is twice as large as the amplitude of the initial perturbation (in the absence of dissipation) (Karpman, 1973). Note that even an infinitely small Reynolds dissipation reduces the maximum amplitude of the perturbation (undular bore) which cannot exceed 1.5 times the amplitude of the initial perturbation in the case of a uniform channel (Chu et al., 1983; Tsuji, 1991).

A strong enough dissipation can prevent infinite amplitude increase and can lead to wave vanishing. For a weaker dissi- 


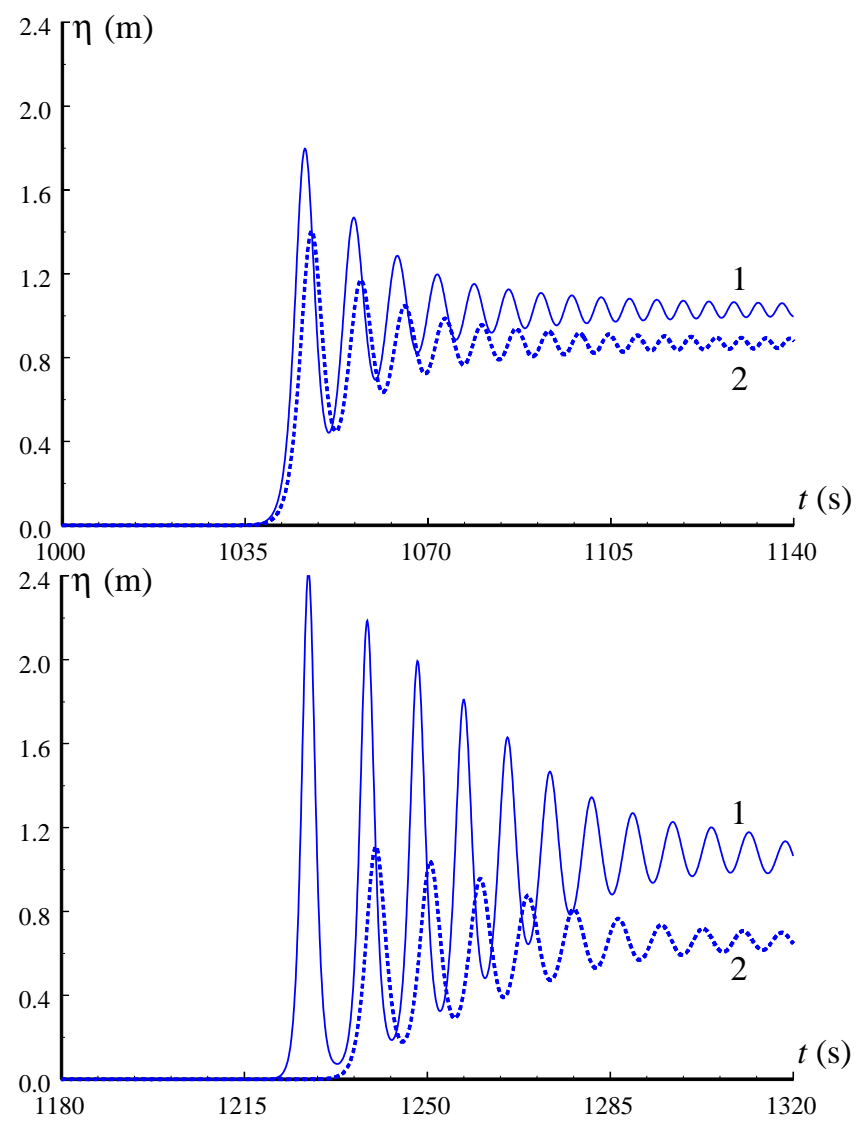

Fig. 10. Bore disintegration into solitons without dissipation (curves 1) and with Chezy dissipation (curves 2). The top panel corresponds to $x=1080 \mathrm{~m}$, while the bottom one is for $x=2930 \mathrm{~m}$.

pation, the amplitude of the soliton can continue to grow due to the decrease of the channel depth or width. As described in Sect. 6, a soliton can propagate with a constant amplitude when the channel inhomogeneity and dissipation act in opposite ways and their parameters are related properly. In our case for the parameters we chose for calculations, the curves labelled 0, 2 and 3 in Fig. 11 reach their maximums and then gradually decrease. We put $v=10^{-2} \mathrm{~m}^{2} / \mathrm{s}, \quad k=10^{-3}$ and implemented three independent runs for models (10)-(12). As one can see, the Chezy dissipation causes the strongest decrease of the soliton amplitude, while the Reynolds dissipation has a very weak effect.

\section{Conclusion}

In this article we have used a generalized $\mathrm{KdV}$ equation to describe long nonlinear waves in channels of rectangular cross section smoothly bending in space and having a variable depth and width. Three different empirical models of dissipation were taken into account and systematically studied.

We studied analytically and numerically the breaking of long nonlinear waves in an inhomogeneous channel, the disintegration of a bore into solitons and the evolution of small

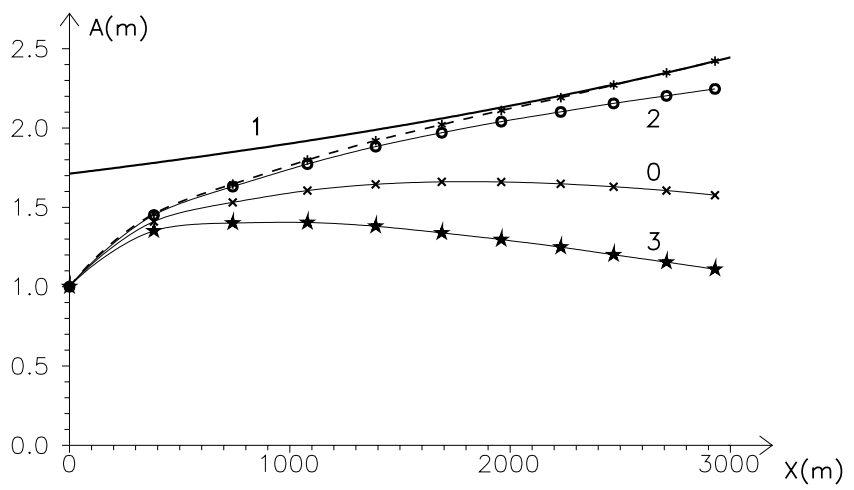

Fig. 11. Maximum amplitude of a step-wise perturbation in the process of its disintegration into solitons. The solid line 1 depicts the theoretical prediction of soliton amplitude dependence on distance when dissipation is neglected. The different numerical calculations shown correspond to no dissipation $(*)$, Rayleigh dissipation $(\times)$, Chezy dissipation $(\star)$ and Reynolds dissipation (०).

initial perturbations into solitons. In particular, we determined the breaking distance of a Riemann wave in a channel of variable depth or width. We showed that a decrease of the depth of the channel induces breaking earlier than the same decrease of the width. In the case when the dispersion effect becomes important the bore decomposes into solitons on a characteristic distance which we estimate and compute precisely when the channel inhomogeneity is known. A constant decrease of the depth or width of the channel reduces this distance as opposed to the homogeneous case. A decrease of the depth causes the decomposition to happen sooner than when the width is decreased. Finally, we have confirmed numerically that once solitons are formed they evolve independently of each other according to nonlinear Green's laws.

Since a reliable model for wave dissipation in channels is not known, we consider different reasonable models and compare their effect. We have examined in detail the three most popular models of dissipation: Rayleigh, Reynolds and Chezy dissipation. All of them give more or less concordant results for the commonly used viscosity parameters. In particluar, we calculated how a small Rayleigh or Chezy dissipation increases breaking distance of Riemann waves. When dispersion is present we showed that these three types of damping reduce the amplitude of the solution and slow down bore decomposition into solitons.

Acknowledgement. The authors thank E. N. Pelinovsky for useful advice and discussions. They also thank the editor and an anonymous referee for remarks which led to a substantial improvement of the article. Y. S. is grateful for the invitation from the INSA de Rouen, France where this work was initiated, and appreciative of the hospitality during his visit in April 2001.

\section{References}

Ablowitz, M. J. and Segur H.: Solitons and the Inverse Scattering Transform, SIAM, Philadelphia, 1981. 
Benilov E. S. and Pelinovsky E. N.: The theory of nonlinear wave propagation in nondispersive media with fluctuating parameters, Sov. Phys. JETP, 67, 98-103, 1988.

Chu C. K., Xiang L. W., and Baransky Y.: Solitary waves induced by boundary motion, Comm. Pure Appl. Math., 36, 495-504, 1983.

Das K. P.: A Korteweg-de Vries equation modified by viscosity for waves in a channel of uniform but arbitrary cross section, Phys. Fluids, 28, 770-775, 1985.

Dyatlov A. I. and Pelinovsky E. N.: Surface wave scattering in a basin with stochastically irregular bottom, Archiwum Hydrotechniki, Poland, 37, 11-17, 1990.

Holloway P. E., Pelinovsky E., Talipova T., and Barnes B.: A nonlinear model of internal tide transformation on the Australian north west shelf, J. Phys. Oceanogr., 27, 871-896, 1997.

Holloway P. E., Pelinovsky E., and Talipova T.: A generalized Korteweg-de Vries model of internal tide transformation in the coastal zone, J. Geophys. Res., 104, 18 333-18 350, 1999.

Grimshaw R.: The solitary wave in water of variable depth, J. Fluid Mech., 42, 639-656, 1970;

Grimshaw R.: The solitary wave in water of variable depth. Part 2. - J. Fluid Mech., 46, 611-622, 1971.

Grimshaw R.: Evolution equations for long, nonlinear internal waves in stratified shear flows, Stud. Appl. Math., 65, 159-188, 1981.

Gurevich, A. V. and Pitaevskii L. P.: Nonstationary structure of collisionless shock wave, Sov. Phys. JETP, 38, 291-297, 1974.

Kakutani T. and Matsuuchi K.: Effect of viscosity on long gravity waves, J. Phys. Soc. Jap., 39, 237-246, 1975.

Karpman V. I.: Nonlinear Waves in Dispersive Media, Moscow, Nauka, (in Russian) 1973, (Engl. transl.: Pergamon Press, Oxford, 1975.)

Khruslov, E. Ya.: Decay of initial steplike perturbation in the Korteweg-de Vries equation, JETP Lett., 21, n. 8, 217-218 (English translation of the Russian journal), 1975. See also: The asymptotic solution of the Cauchy problem for the KdV equation with steplike initial data, Matematicheskii Sbornik, 99, n. 2, 261-281 (in Russian) 1976.

Leontjev I. O.: Dynamics of Surf Zone, Inst of Oceanology, Russ. Acad. Sci., Moscow, 184, (in Russian) 1989.

MacKraken D. and Dorn Y.: Numerical Methods and FORTRAN Programming, John Willey \& Sons, New York, 1975.

Mazumber N. C. and Bose S.: Formation and propagation of tidal bore, J. Wtrways., Port, Coast. and Oc. Engrg., 167-175, 1995.

Mei C. C.: The Applied Dynamics of Ocean Surface Waves, John Wiley \& Sons, New York, 1983.

Merchant T. R. and Smyth N. F.: Initial-boundary problems for the Korteweg-de Vries equations, IMA J. Appl. Math., 47, 247-264, 1991.

Merchant T. R. and Smyth N. F.: The initial boundary problem for the Korteweg-de Vries equation on negative quarter-plane, Proc. R. Soc. Lond. A, 458, 857-871, 2002.

Miles J. W.: Korteweg-de Vries equation modified by viscosity, Phys. Fluids, 19, 1063, 1975.

Miles J. W.: Damping of weakly nonlinear shallow-water waves, J. Fluid Mech., 76, 251-257, 1976.

Myint, S. and Grimshaw, R.: The modulation of nonlinear periodic wavetrains by dissipative terms in the Korteweg-de Vries equation, Wave Motion, 22, 215-238, 1995.

Osborne A. R.: The inverse scattering transform: tools for the nonlinear Fourier analysis and filtering of ocean surface waves, Chaos, solitons and fractals, 5, 2623-2637, 1995.

Ostrovsky L. A.: Approximate methods in the theory of nonlinear waves, Radiophysics and Quantum Electronics, 17, 344-360, 1974.

Ostrovsky L. A. and Pelinovsky E. N.: Wave transformation on the surface of fluid of variable depth, Sov. Phys. Izvestia. Atmospheric and Oceanic Physics, 6, 552-555, 1970.

Ostrovsky L. A. and Pelinovsky E. N.: Refraction of nonlinear sea waves in the coastal zone, Sov. Phys. Izvestia. Atmospheric and Oceanic Physics, 11, 68-74, 1975.

Ott E. and Sudan R. N.: Damping of solitary waves, Phys. Fluids, $13,1432-1434,1970$

Pelinovsky E. N.: Hydrodynamics of Tsunami Waves, IPF RAN, Nizhny Novgorod, (in Russian) 1996.

Pelinovsky E. N.: On the absorption of nonlinear waves in dispersive media, Sov. Phys. J. Appl. Mech. Tech. Phys., 68-71, 1971.

Pelinovsky E. N. and Stepanyants Yu. A.: On the estimation of the time of solitons creations from the initial perturbations in the framework of Korteweg-de Vries equation, Izv. VUZov, Radiofizika, 24, 908-911, (in Russian) 1981.

Pelinovsky E. N., Stepanyants Yu. A., and Talipova T. G.: Nonlinear dispersion model of sea waves in the coastal zone, J. Korean Soc. Coastal Ocean Eng., 5, 307-317, 1993.

Stepanyants Yu. A.: Relationship between kinetic and potential energies in internal waves in the presence of shear currents, Sov. Phys. Izvestia. Atmospheric and Oceanic Physics, 21, 518-520, 1985.

Tsuji Y., Yanuma T., Murata I., and Fujiwara C.: Tsunami ascending in rivers as an undular bore, Natural Hazards, 4, 257-266, 1991.

Whitham G. B.: Linear and Nonlinear Waves, J. Wiley \& Sons, 636, 1974.

$\mathrm{Wu}$ Y. H. and Tian J.-W.: Mathematical analysis of long-wave breaking on open channels with bottom friction, Ocean Eng., 26, 187-201, 2000.

Voltsinger N. E., Klevany K. A., and Pelinovsky E. N. Long-Wave Dynamics of the Coastal Zone., Gidrometeoizdat, Leningrad, $271,1989$. 